\title{
Scattering and dip angle decomposition based on subsurface offset extended wave-equation migration
}

\author{
Raanan Dafni ${ }^{1}$ and William W. Symes ${ }^{1}$
}

\begin{abstract}
An angle-dependent reflection coefficient is recovered by seismic migration in the angle domain. We have developed a postmigration technique for computing scattering and dip angle common-image gathers (CIGs) from seismic images, extended by the subsurface offset, based on wave-equation migration methods. Our methodology suggests a system of Radon transform operators by introducing local transform relations between the subsurface offset image and the angle-domain components. In addition to the commonly used decomposition of the scattering angle, the methodology associates the wave-equation migration with dip-domain images as well. The same postmigration subsurface offset image is used to decompose scattering and dip angle CIGs individually or to decompose a multiangle CIG by showing simultaneously both angles on the gather's axis. We
\end{abstract}

show that the dip-angle response of seismic reflections is a spot-like signature, focused at the specular dip of the subsurface reflector. It differs from the well-studied smile-like response usually associated with reflections in the dip domain. The contradiction is clarified by the nature of the subsurface offset extension, and by emphasizing that the angles are decomposed from the subsurface offset image after the imaging condition, without directly involving the propagating incident and scattered wavefields. Several synthetic and field data tests proved the robustness of our decomposition technique, by handling various subsurface models, including seismic diffractions. It is our belief that dip-angle information, decomposed by wave-equation migration, would have a great impact in making the scattering-angle reflection coefficient more reliable and noise free, in addition to the acceleration of wave-equation inversion methods.

\section{INTRODUCTION}

Seismic depth migration in the angle domain has become a common method for imaging and studying the interior of the earth in recent years. In the angle domain, common-image gathers (CIGs) collect energy from the seismic data that have been scattered over a specific angular direction. We will refer here to these gathers as angle-domain CIGs (ADCIGs). ADCIGs present prestack angledependent reflectivity, which is essential for reliable and accurate image interpretation, migration velocity analysis, and amplitude variation with angle analysis. Over the years, many authors emphasized the importance of using angle-domain imaging methods according to the subsurface angle of scattering, rather than the universally used acquisition offset (ten Kroode et al., 1994; Nolan and Symes, 1996; Xu et al., 2001; Brandsberg-Dahl et al., 2003; Biondi and Symes, 2004; Biondi and Tisserant, 2004; Koren and
Ravve, 2011). It was argued that angle-domain imaging can better handle imaging tasks over complex geologic areas where the wavefield includes multipathing. An event in an ADCIG uniquely determines a ray pair, which in turn uniquely locates the reflector. Therefore, mapping the seismic data to the angle domain is unique and naturally unfolds multivalued raypaths (Prucha et al., 1999).

Migration in the angle domain is usually referred to the asymptotic integral formulation of the linearized inverse scattering problem, introduced by Beylkin (1985). This Born-type inversion is based on the Beylkin determinant, which transforms the integration variables from the seismic experiment coordinates on the acquisition surface to the local angle domain (LAD) in the subsurface (Bleistein, 1987). The oscillatory kernel of the inversion's integral formulation is related via a $1 \mathrm{D}$ Fourier transform to the generalized Radon transform (GRT). Beylkin (1985) and Miller et al. (1987) emphasize the connection between the scattering problem and

\footnotetext{
Manuscript received by the Editor 3 October 2015; revised manuscript received 30 December 2015; published online 27 April 2016.

${ }^{1}$ Rice University, Department of Computational and Applied Mathematics, Houston, Texas, USA. E-mail: raanandaf@yahoo.com; symes@caam.rice.edu.

(C) 2016 Society of Exploration Geophysicists. All rights reserved.
} 
the Radon transform, and they cast their solution to the linearized inverse scattering problem as an inverse GRT. They describe their formulation as "migration by inversion of a GRT." The basis of their approach is that integrating the seismic data over isochron surfaces by angle-domain variables recovers an image of the scattering potential (i.e., reflectivity). The idea was to back project the seismic data to the image space by a weighted diffraction stack operator to reconstruct the scattering potential.

In this paper, another relationship between migration in the angle domain and the Radon transform will be followed, although it will be formulated in the postmigration image space rather than in the data space. Migrated seismic data in the subsurface offset domain will be used instead of the acquired surface offset data. We will distinguish between two independent angular systems usually involved in angle-domain migrations to generate ADCIGs: The scattering-angle system and the dip-angle system. The first one provides a natural access to migration velocity and amplitude analysis according to the scattering angle at the local reflecting surface, whereas the latter holds essential information about the subsurface structure and its related specular direction. Incorporating these two angle systems together in the process of seismic imaging, velocity determination or amplitude analysis has been shown as crucial in terms of efficiency and accuracy (Audebert et al., 2003; Brandsberg-Dahl et al., 2003; Reshef and Rueger, 2008; Koren and Ravve, 2011; Dafni and Reshef, 2014, 2015).

ADCIGs can be computed by either using Kirchhoff or waveequation migration methods. Both cases share the same characteristic of describing an angle-dependent reflectivity at the subsurface reflector. However, Kirchhoff ADCIGs might be contaminated with significant artifacts due to imaging by the asymptotic approximation of the ray theory, especially in areas with structural complexity or when strong lateral velocity variations are present (Stolk and Symes, 2004). Therefore, wave-equation migration methods are considered as a natural choice for producing ADCIGs, although higher computational cost is usually involved.

Generally speaking, the methods for calculating ADCIGs based on wave-equation migration fall into two main categories. The first is related to those methods applied by either shot-profile or shotgeophone migration techniques in the data space (de Bruin et al., 1990; Prucha et al., 1999; Mosher and Foster, 2000; Wu and Chen, 2006; Yoon and Marfurt, 2006). These methods evaluate the ADCIGs by slant stacks of the downward-continued wavefield at each time/depth step prior to the imaging condition. Therefore, they tend to be computationally expensive. They usually provide high-angular resolution images, but when the wavefield becomes complicated, due to complex subsurface geology, accuracy issues may arise. The second group of methods includes those applied in the image space after the imaging condition (Rickett and Sava, 2002; Sava and Fomel, 2003; Biondi and Symes, 2004). They do not involve the wavefield anymore, but the prestack image. Therefore, they are also considered as postmigration techniques. The prestack image is usually obtained by an extended imaging condition, which captures the image at different space or time correlation lags (i.e., subsurface offset or time shift; Sava and Vasconcelos, 2011). The ability to decompose explicitly the scattering and dip angles through a postmigration operation makes these methods relatively convenient and robust, although the migration implementation by the extended imaging condition usually increases the computational cost due to the extra dimensions of the image.
Due to their extensive use, tremendous effort was given in recent years to derive, compute, and study scattering-angle ADCIGs in relation with wave-equation migration. Jin et al. (2014) introduce an elaborated overview of the main techniques available, and evenly compared between them. However, dip-angle ADCIGs based on wave equation migration remained nearly unstudied. $\mathrm{Wu}$ and Chen (2003, 2006) evaluate the dip-angle response out of a local image matrix computed by beamlet wavefield decomposition. Browaeys (2008) suggests decomposing the dip angles out of their relation with the vertical and horizontal components of the subsurface offset. Li et al. (2012) use a directional wavefield decomposition to compute reverse time migration dip-angle ADCIGs, and use them to eliminate illumination artifacts in the image.

Introducing a robust wave-based dip-angle decomposition technique is one of the main goals this study is after. The novelty of our paper is the deduced relation between the local dip angle of reflection and the subsurface offset extended image. It is derived explicitly from the wave equation, and it involves the computation of a uniaxial image extension (the horizontal subsurface offset solely). Because this extension is commonly used for scattering-angle decomposition as well, our method provides the two types of ADCIGs out of the same uniaxial image extension. It is our belief that the dip-angle domain should be as promising for wave-equation migration methods as it is for Kirchhoff migration methods.

In the following, a set of image space techniques is proposed for decomposing ADCIGs derived from subsurface offset extended wave-equation migration. We start by introducing some new insights regarding the image behavior in the subsurface offset domain. An intriguing analogy between the acquisition and the subsurface offsets is deduced. Then, a suite of Radon transform formulations is suggested to transform the subsurface offset image extension into the scattering-angle and the dip-angle image systems. We base our techniques on the approach proposed by Sava and Fomel (2003) for scattering-angle decomposition, and expand it to also enable dipangle decomposition. It is shown that once scattering and dip dependent images are formulated, the derivation of a multiangle image gather is within reach. This hybrid gather represents the image by the scattering and dip components simultaneously, in the same style proposed by Dafni and Reshef (2012) and similar to the approach suggested by $\mathrm{Wu}$ and Chen (2006). We also discuss the unique nature the scattering and dip angles have, once they are decomposed according to the subsurface offset, and we compare our results with Kirchhoff migration techniques.

\section{SUBSURFACE OFFSET IMAGE EXTENSION}

Prestack migration operators can be described as the adjoint of extended Born-type modeling operators, after extending the definition of the reflectivity to depend on more degrees of freedom (Symes, 2008; Stolk et al., 2009). It allows migration velocity and amplitude analysis to exploit the redundancy in the seismic data by using image gathers in the extended domain. One conventional and natural choice to extend the reflectivity is by the subsurface offset, as introduced by Claerbout (1985) in the framework of survey-sinking migration. It is defined as the Cartesian offset vector connecting the sunken shot and receiver in the subsurface (see Figure $1 \mathrm{~b}$ ), and it involves an action at a distance between the incident and scattered wavefields. The image $I(\mathbf{x}, \mathbf{h})$, extended by the subsurface half-offset $\mathbf{h}$ takes the form 


$$
\begin{aligned}
I(\mathbf{x}, \mathbf{h})= & \int d \mathbf{x}_{\mathbf{r}} \int d \mathbf{x}_{\mathbf{s}} \int d t \frac{\partial^{2}}{\partial t^{2}} D\left(\mathbf{x}_{\mathbf{r}}, t ; \mathbf{x}_{\mathbf{s}}\right) \\
& \times \int d \tau G\left(\mathbf{x}+\mathbf{h}, t-\tau ; \mathbf{x}_{\mathbf{r}}\right) G\left(\mathbf{x}-\mathbf{h}, \tau ; \mathbf{x}_{\mathbf{s}}\right),
\end{aligned}
$$

where $G(\mathbf{x}, t)$ is the Green's function, $D\left(\mathbf{x}_{\mathbf{r}}, t ; \mathbf{x}_{\mathbf{s}}\right)$ stands for the seismic data, and $\tau$ is the migration time (Stolk et al., 2009). For a perfectly known velocity model, significant action will take place only at zero subsurface offset (i.e., the physical offset), and therefore, the extended image will be perfectly focused at zero offset. Likewise, incorrect velocity will defocus the image and may produce a fake reflector image at nonzero offset (i.e., nonphysical offset). The subsurface offset extension can be restricted by setting one of its spatial coordinates to zero. In this study, the depth coordinate will be restricted to zero, making the extension a function of the horizontal components only: $\mathbf{h}=\left(h_{x}, h_{y}, 0\right)$. In the 2D case, according to this restriction, the subsurface offset becomes a scalar in equation 1 . The restricted extension to the horizontal direction solely becomes singular at the limit, where vertical reflectors are imaged (Biondi and Symes, 2004). This limits the use of equation 1 (subjected to the restriction) when nearly vertical reflectors are present. In such a case, the horizontal subsurface offset extension is expected to degenerate gradually, and the focusing at zero offset is expected to blur. Having said that, steep reflectors, which are not nearly vertical, are still valid and would be properly handled.

In signal processing, an impulse response refers to the response of a dynamic system to a brief input signal. In relation to seismic imaging, it is the response of the image space to the migration of a single data trace (Yilmaz, 2001). An extended impulse response can be calculated in the subsurface offset domain according to the extended migration operator in equation 1 . Full-analytic derivation for this extended impulse response is given in Appendix A, whereas the main results are summarized next. For simplicity, we study here the $2 \mathrm{D}$ case, where the acquisition and subsurface offsets act as scalars.

For a delta-function reflection at depth $z_{o}$ in a known and homogeneous medium, the subsurface offset extended impulse response has an elliptic form in $z-x$ image sections (constant $h$ ):

$$
\frac{\left(x-x_{m}\right)^{2}}{\left(z_{0}^{2}+H^{2}\right)}+\frac{z^{2}}{\left(z_{0}^{2}+H^{2}\right)-(h-H)^{2}}=1,
$$

where $H$ and $h$ are the acquisition and subsurface half-offsets, respectively, and $x_{m}$ is the midpoint location of the data trace. This equation is illustrated in Figure 2 for two scenarios: (1) The response of a zero acquisition offset trace $(H=0 \mathrm{~m})$ is shown in Figure 2a, and (2) the response of a finite-acquisition offset trace $(H=1000 \mathrm{~m})$ is shown in Figure 2b. In both cases, $z_{o}$ is set to $2000 \mathrm{~m}$ (indicated by the dashed black line), and $x_{m}$ is $4000 \mathrm{~m}$. The elliptic response at zero and finite subsurface offset image sections ( $h=0 \mathrm{~m}$ and $h=-1000 \mathrm{~m}$ ) are folded together in the figures. In each of the two scenarios, the ellipses represent isochron curves of equal shot-receiver traveltime. The dashed gray lines indicate that the same traveltime is conserved also in the extended case, when the subsurface offset is not zero. Notice that the focal distance of the ellipse is defined by the difference $|h-H|$. Figure 3 shows the migration results for the same two scenarios, by computing the extended image according to the operator in equation 1 . The images in Figure $3 \mathrm{a}$ and $3 \mathrm{~b}$ correspond to the migration of acquisition offsets $H=0$ and $1000 \mathrm{~m}$, respectively, where the $h=0 \mathrm{~m}$ and the $h=-1000 \mathrm{~m}$ image sections are shown on top and at the bottom, respectively. All four image sections perfectly follow the analytic elliptic formula of equation 2 .

An interesting comparison is made between the nature of the acquisition and subsurface offsets. By setting the acquisition offset to zero $(H=0 \mathrm{~m})$, and equivalently by setting the subsurface offset to zero $(h=0 \mathrm{~m})$, we obtain equations 3 and 4 , respectively:

$$
\begin{gathered}
\frac{\left(x-x_{m}\right)^{2}}{z_{0}^{2}}+\frac{z^{2}}{\left(z_{0}^{2}-h^{2}\right)}=1, \\
\frac{\left(x-x_{m}\right)^{2}}{\left(z_{0}^{2}+H^{2}\right)}+\frac{z^{2}}{z_{0}^{2}}=1 .
\end{gathered}
$$

Table 1 summarizes the geometric comparison between the acquisition and the subsurface offset ellipses of equations 4 and 3 . Because both offsets have the same meaning of shot-receiver distance (although one is on the recording surface and the other is sunken), there is a strong correlation in their nature of response in the image space. The ellipses focal distance is represented by the offset, with the shot and receiver coordinates (whether sunken or not) defining the focal points. The semimajor axis of one ellipse resembles the semiminor axis of the other, and vice versa. Moreover, the eccentricity is also equivalent because it is represented by the sine of the scattering angle (denoted here by $\gamma$ ) at zero dip. Table 1 also provides the horizontal and vertical stretch factors a)

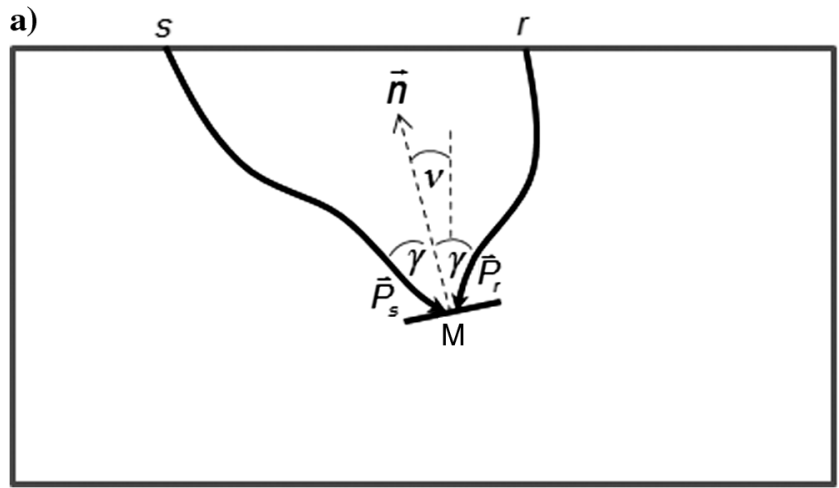

b) $s$

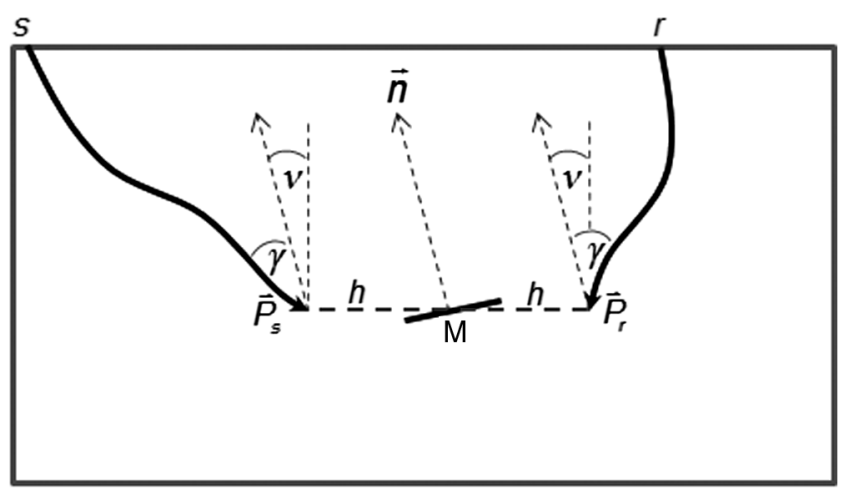

Figure 1. The interaction between the incident and scattered plane waves (a) at zero offset and (b) at finite offset generates an image at the subsurface midpoint M. The scattering and dip angles are defined accordingly, where the interaction takes place. 
a)

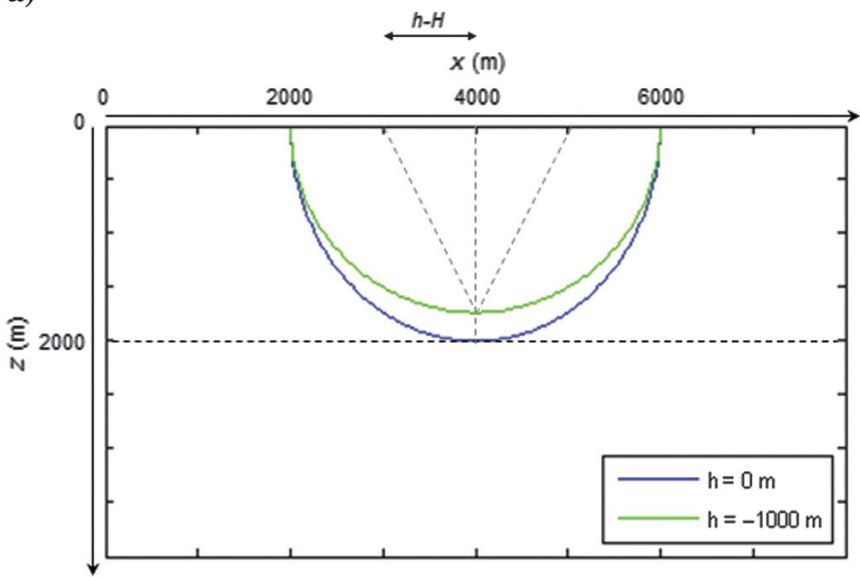

b)
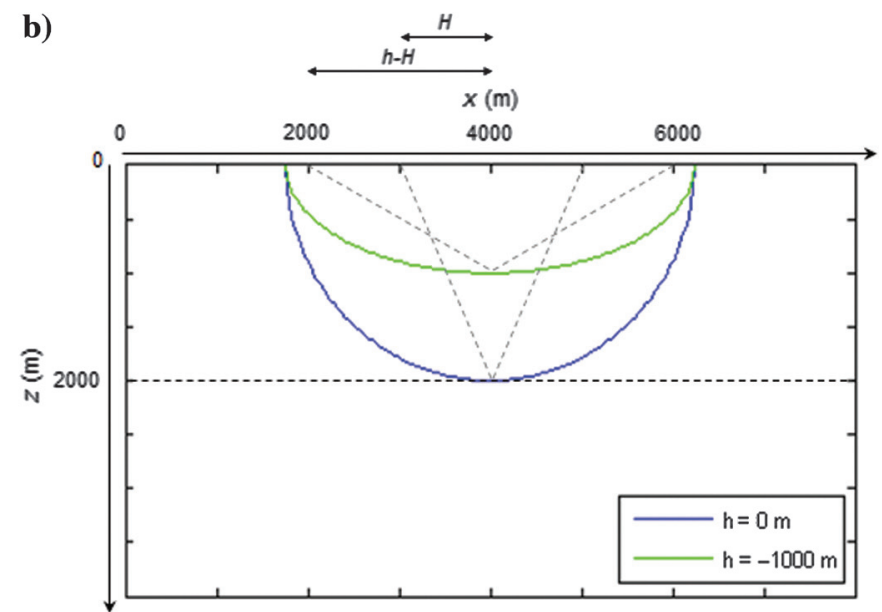

Figure 2. Extended impulse response in the $z-x$ domain, generated by (a) $0 \mathrm{~m}$ acquisition offset data trace and (b) $1000 \mathrm{~m}$ acquisition offset data trace. The elliptic response of 0 and $-1000 \mathrm{~m}$ subsurface offsets are folded together, representing isochron curves of equal shot-receiver traveltime. The ellipse's focal distance is defined by the difference between the acquisition and subsurface offsets.

a)
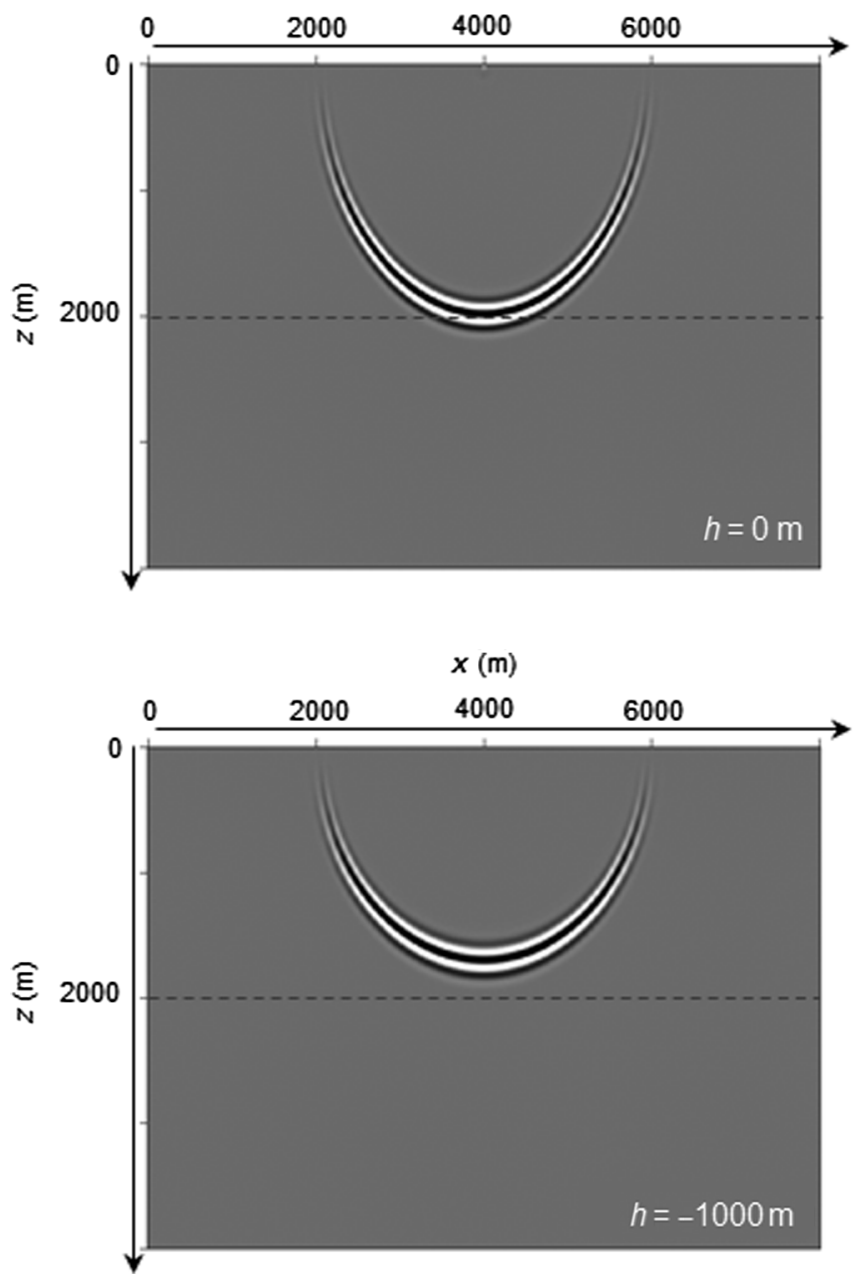

b)
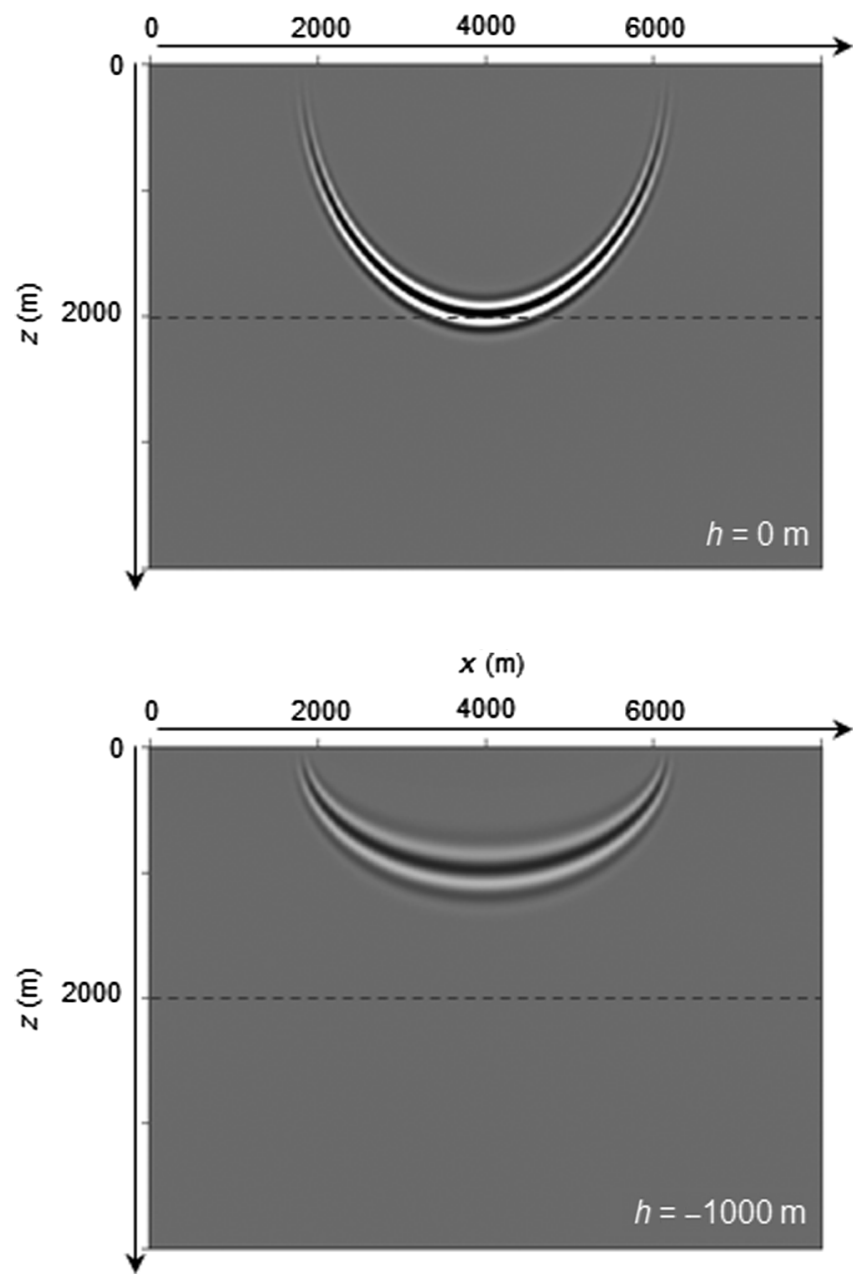

Figure 3. Extended impulse response in the $z-x$ domain. The images corresponding to the migration of (a) $0 \mathrm{~m}$ acquisition offset and (b) $1000 \mathrm{~m}$ acquisition offset are presented. The 0 and $-1000 \mathrm{~m}$ subsurface offset image sections are shown on top and at the bottom, respectively. 
to transform the ellipses into circles. Conceptually, this interesting symmetry allows the reconstruction of one type of offset from the other by the use of the stretch factors in Table 1. For example, as illustrated in Figure 4, the accumulative impulse response of all acquisition offsets, defining an entire CMP gather in the data space, can be reconstructed in the extended image space, by migrating only a single data trace. A zero-offset data trace (see Figure 4a) is migrated into the extended subsurface offset domain (see Figure 4b). Then, the horizontal and vertical axes are stretched to transform from equations 3 to 4 , and all offsets are stacked. The result is the reconstructed image in Figure 4c, which represents the accumulative impulse response of the entire CMP gather.

Rearranging equation 2 to represent the extended impulse response in the $z-h$ image gather domain (constant $x$ ), yields the following elliptic equation:

$$
\frac{(h-H)^{2}}{\left(z_{0}^{2}+H^{2}\right)}+\frac{z^{2}}{\left(z_{0}^{2}+H^{2}\right)-\Delta x^{2}}=1,
$$

Table 1. Comparison between the geometrical properties associated with the acquisition offset ellipse in equation 4 , and the subsurface offset ellipse in equation 3.

\begin{tabular}{lcc}
\hline & $\begin{array}{c}\text { Acquisition offset } \\
\text { ellipse }\end{array}$ & $\begin{array}{c}\text { Subsurface offset } \\
\text { ellipse }\end{array}$ \\
\hline $\begin{array}{l}\text { Focal distance } \\
\text { Semimajor axis }\end{array}$ & $H$ & $h$ \\
Semiminor axis & $\sqrt{z_{0}^{2}+H^{2}}$ & $z_{0}$ \\
Eccentricity & $z_{0}$ & $\sqrt{z_{0}^{2}-h^{2}}$ \\
$\begin{array}{l}\text { Horizontal stretch } \\
\text { factor }\end{array}$ & $\sqrt{\frac{H^{2}}{z_{0}^{2}+H^{2}}}=\sin \gamma_{(h=0)}$ & $\sqrt{\frac{h^{2}}{z_{0}^{2}}}=\sin \gamma_{(H=0)}$ \\
Vertical stretch factor & $S_{x}=\sqrt{1+\frac{H^{2}}{z_{0}^{2}}}$ & - \\
\hline
\end{tabular}

where $\Delta x=x-x_{m}$ is the horizontal distance between the image point and the data midpoint (i.e., imaging aperture). Because a homogeneous medium is assumed at this part of the paper, the imaging aperture is directly related to the structural dip of the seismic event. In the special case in which a zero-dip reflector is being imaged, the image location $x$ coincides with the data midpoint $x_{m}$ and the aperture vanishes $(\Delta x=0)$. In such a case, the ellipse in equation 5 becomes a circle:

$$
\frac{(h-H)^{2}}{\left(z_{0}^{2}+H^{2}\right)}+\frac{z^{2}}{\left(z_{0}^{2}+H^{2}\right)}=1 .
$$

The radius of the circle is defined by the denominator on the left side according to the conserved traveltime of the response. The center of the circle is shifted horizontally along the $h$-axis by the acquisition offset $H$. Equation 6 is illustrated in Figure 5 for the same two scenarios mentioned above: the response of a zero acquisition offset trace $(H=0 \mathrm{~m})$ and the response of a finite acquisition offset trace $(H=1000 \mathrm{~m})$. The two circles share the same intersection point with the vertical axis of the gather, which represents the imaging depth of the reflector $z_{o}$ at zero subsurface offset (indicated by the dashed black cross).

Figure 6 shows the migration results in this case, which perfectly follow the analytic circular formula of equation 6 . As more and more data traces are accumulated through the migration, a constructive interference occurs at the imaging depth $z_{o}$ and at the zero subsurface offset trace, where all the circular responses intersect. Moreover, destructive interference takes place at nonzero subsurface offset traces, where the circular responses come out of phase. As a result, the final reflection image becomes focused at zero subsurface offset. However because the acquisition geometry is always bounded by a finite-maximum offset $\left(H_{\max }\right)$, the destructive interference away from the zero subsurface offset will always leave some remnant nondestructive part of energy that contaminates the image. This emphasizes another interesting relation between the acquisition and subsurface offsets. The image is contaminated with kinematic artifacts away from the zero subsurface offset, related to the truncation of the data by a maximum acquisition offset.

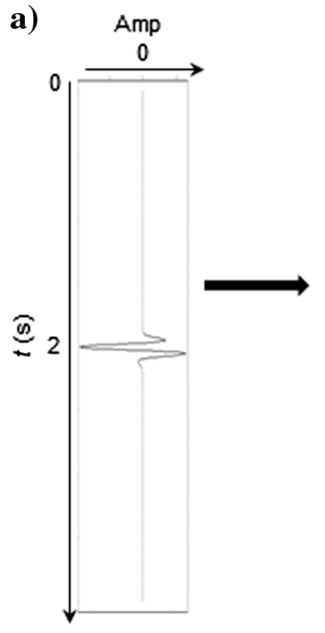

b)

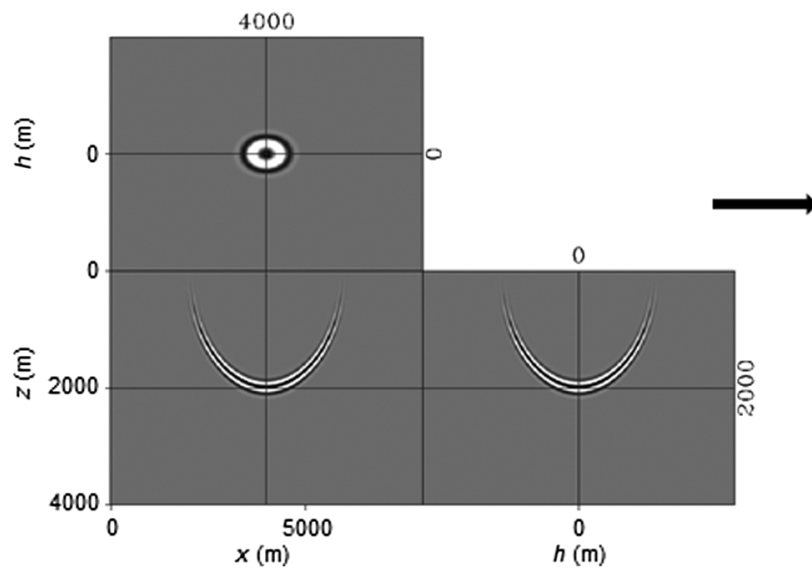

c)

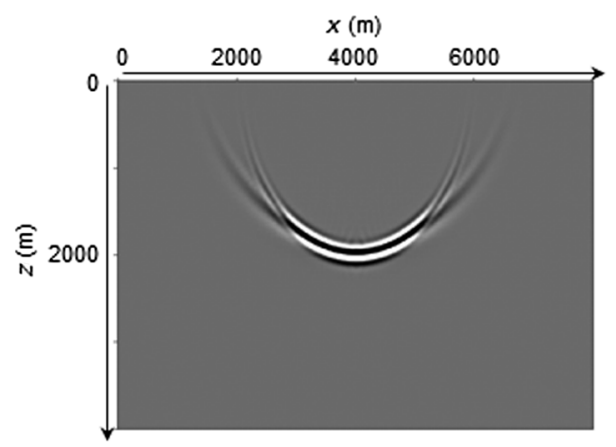

Figure 4. The construction of the accumulated impulse response of an entire CMP gather, by stretching and folding an extended impulse response of a single data trace. (a) The zero-offset data trace, (b) the extended impulse response in the subsurface offset domain (a 3D view), and (c) the nonextended accumulated impulse response of the CMP gather. 
We can conclude this part of our study by considering prestack migration in the subsurface offset domain as a superposition of extended impulse responses, made by individual data traces. Mulder (2014) derives a similar analytic formula to the one we proposed in equation 6 . His approach was based on a stationary phase analysis in the high-frequency limit, for an unknown but homogeneous medium. His claim, that the only portion of significant migrated energy left at nonzero subsurface offsets is related to the maximum offset of acquisition, is in line with our conclusion about the truncation of the impulse response superposition.

\section{SUBSURFACE OFFSET RELATIONS WITH THE ANGLE DOMAIN}

Determining the direction of scattering in any angle-domain imaging system involves a local measurement in the subsurface, where incident and scattered wavefields interacts. The locality of the measurement allows the wavefields to be described as local plane waves (or rays) by high-frequency asymptotics. The appearance of a continuous reflection event in the seismic data is equivalent to the presence of incident and scattered plane-wave components, interacting at an image point and sharing the same phase space coordinates as the subsurface reflector (i.e., obeying Snell's law; Stolk et al., 2009). The phase space coordinates are usually referred to the traveltime gradient or the slowness vector of the incident and scattered waves: $\nabla T_{s}=\mathbf{P}_{\mathbf{s}}\left(t_{s}, \mathbf{x}\right)$ and $\nabla T_{r}=\mathbf{P}_{\mathbf{r}}\left(t_{r}, \mathbf{x}\right)$, respectively. They indicate the direction of propagation as illustrated in Figure 1a. Extending the migration operator by the subsurface offset, as introduced in equation 1 , also extends the definition of the slowness vectors to account the additional dimension. The slownesses take the form $\mathbf{P}_{\mathbf{s}}\left(t_{s}, \mathbf{x}-\mathbf{h}\right)$ and $\mathbf{P}_{\mathbf{r}}\left(t_{r}, \mathbf{x}+\mathbf{h}\right)$, respectively. They no longer interact at the same image point and construct an image at the subsurface midpoint coordinate, distinct away horizontally by a factor of $h$ from each one of them as shown in Figure 1b.

In this study, we are after an angle-domain system involving the scattering and the dip angles. We follow the LAD imaging system introduced by Koren and Ravve (2011) and Ravve and Koren (2011). According to their proposal, a set of four angles is required to determine the LAD imaging system in the general $3 \mathrm{D}$ case. Two

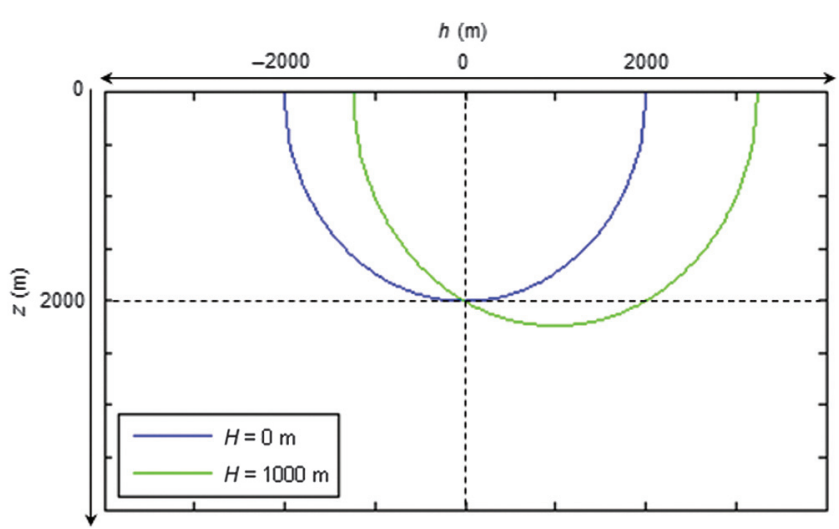

Figure 5. Extended impulse response in the $z-h$ domain. The responses of 0 and $1000 \mathrm{~m}$ acquisition offset data traces are illustrated. Circular responses are accumulated in the subsurface offset CIG, sharing the same intersection point with the vertical axis when the medium velocity is known. angles are associated with the dip angle $\nu_{1}$ and the dip azimuth $\nu_{2}$ of the interacting plane waves, defined by the normal direction. The other two angles are the scattering angle and scattering-azimuth, representing the opening angle $\gamma_{1}$ between the interacting plane waves and its azimuth $\gamma_{2}$. In the azimuth-free 2D case, only two angles, namely the dip angle $\nu$ and the scattering angle $\gamma$, comprise the LAD system as illustrated in Figure $1 \mathrm{a}$ and $1 \mathrm{~b}$. Notice that when the subsurface offset is finite, the interaction between the incident and scattered plane waves does not share the same image point. Moreover, the angles are measured individually at two image points, where the plane waves interact, away from the subsurface midpoint, where the image is constructed (see Figure 1a and 1b). Hence, the angle decomposition at zero and nonzero subsurface offsets is fundamentally different in meaning. Defocusing the image away from the zero subsurface offset, due to erroneous migration velocity, might lead to ambiguous angle-domain decomposition and analysis (Bartana et al., 2006; Montel and Lambare, 2013).

Sava and Fomel (2003) derive the 2D case relation between the LAD system and the plane-wave slowness vectors in the extended image space as follows:

$$
\begin{aligned}
& \nabla T_{s}=\mathbf{P}_{\mathbf{s}}\left(t_{s}, \mathbf{x}-h\right)=\frac{\sin (\nu-\gamma) \mathbf{i}+\cos (\nu-\gamma) \mathbf{j}}{V(\mathbf{x}-h)}, \\
& \nabla T_{r}=\mathbf{P}_{r}\left(t_{r}, \mathbf{x}+h\right)=\frac{\sin (\nu+\gamma) \mathbf{i}+\cos (\nu+\gamma) \mathbf{j}}{V(\mathbf{x}+h)},
\end{aligned}
$$

where $\mathbf{i}$ and $\mathbf{j}$ are the unit vectors along the $x$ - and $z$-directions, respectively, and $V$ is the velocity. Note that the slowness vectors in equation 7 are calculated in the subsurface, and require by definition that the velocity will not vary locally at the point of scattering. Otherwise, there are no rays, whose slowness vectors define angles. In their paper, Sava and Fomel (2003) recast equation 7 to represent the gradient of the total traveltime with respect to the extended image coordinates:

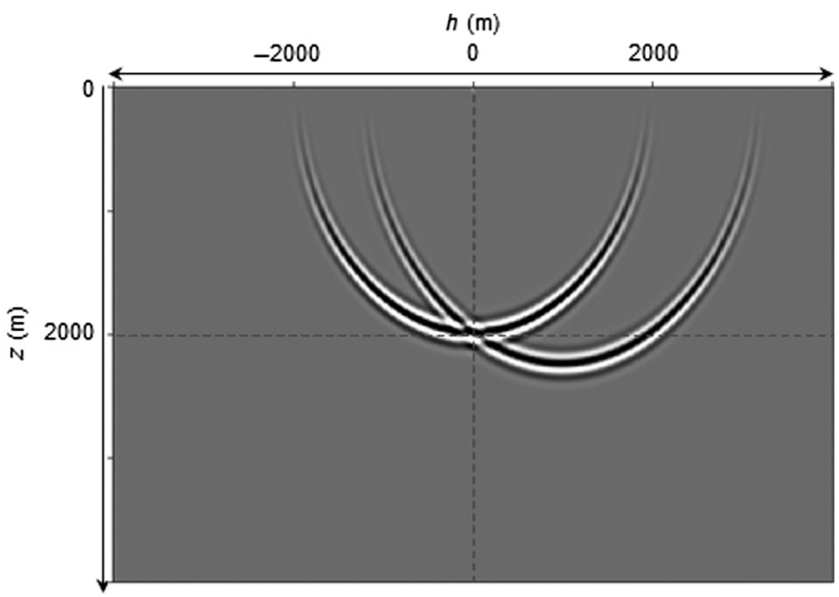

Figure 6. Extended impulse response in the $z-h$ domain. The image corresponding to the migration of 0 and $1000 \mathrm{~m}$ acquisition offset data traces is shown. Circular responses are accumulated in the subsurface offset CIG, sharing the same intersection point with the vertical axis when the medium velocity is known. 


$$
\begin{aligned}
\frac{\partial}{\partial x}\left(T_{r}+T_{s}\right) & =\left(\mathbf{P}_{r}+\mathbf{P}_{s}\right) \mathbf{i}=\frac{2 \sin \nu \cos \gamma}{V(x)}, \\
\frac{\partial}{\partial h}\left(T_{r}+T_{s}\right) & =\left(\mathbf{P}_{r}-\mathbf{P}_{s}\right) \mathbf{i}=\frac{2 \cos \nu \sin \gamma}{V(x)}, \\
\frac{\partial}{\partial z}\left(T_{r}+T_{s}\right) & =\left(\mathbf{P}_{r}+\mathbf{P}_{s}\right) \mathbf{j}=\frac{2 \cos \nu \cos \gamma}{V(x)} .
\end{aligned}
$$

It should be emphasized that the relation between the ray quantities and the image wave vectors embodied in equation 8 holds only for the subsurface offset $h=0$. This is the physical image section, where the image is focused due to correct migration velocity. Hence, equation 8 is restricted to provide true angle quantities only when the velocity is perfectly known. Otherwise, the image defocuses to nonphysical subsurface offsets $(h !=0)$, and the use of equation 8 leads to incorrect angle quantities that differ in their meaning from the true ones (Bartana et al., 2006).

From equation 8 , the following relationship with the scattering angles is obtained:

$$
-\left.\frac{\partial z}{\partial h}\right|_{t, x}=\tan \gamma=-\frac{k_{h}}{k_{z}} .
$$

The left side implies a relation between the scattering angles and constant slope trajectories in the subsurface offset extended image space. The right side presents an equivalent relation, in Fourier domain, according to the ratio between the subsurface offset wavenumber $k_{h}$ and the vertical wavenumber $k_{z}$.

The differential relations in equation 8 can also provide an analogous expression with regards to the dip angles:

$$
-\left.\frac{\partial z}{\partial x}\right|_{t, h}=\tan \nu=-\frac{k_{x}}{k_{z}} .
$$

The dip angles are associated with constant slope trajectories, at constant $h$ image sections, whereas in the Fourier domain, they are associated with the ratio between the horizontal wavenumber $k_{x}$ and the vertical wavenumber $k_{z}$.

The angle-domain relations with the horizontal subsurface offset are extended to 3D by computing a 2D subsurface offset extension: $\mathbf{h}=\left(h_{x}, h_{y}, 0\right)$, in addition to the three physical dimensions of the image: $\mathbf{x}=(x, y, z)$. Equation 8 is extended accordingly to take the form

$$
\begin{aligned}
\frac{\partial}{\partial x}\left(T_{r}+T_{s}\right) & =\frac{2 \sin \nu_{1} \cos \nu_{2} \cos \gamma_{1}}{V(x)} ; \\
\frac{\partial}{\partial y}\left(T_{r}+T_{s}\right) & =\frac{2 \sin \nu_{1} \sin \nu_{2} \cos \gamma_{1}}{V(x)}, \\
\frac{\partial}{\partial h_{x}}\left(T_{r}+T_{s}\right) & =\frac{2 \cos \nu_{1} \sin \gamma_{1} \cos \gamma_{2}}{V(x)} ; \\
\frac{\partial}{\partial h_{y}}\left(T_{r}+T_{s}\right) & =\frac{2 \cos \nu_{1} \sin \gamma_{1} \sin \gamma_{2}}{V(x)}, \\
\frac{\partial}{\partial z}\left(T_{r}+T_{s}\right) & =\frac{2 \cos \nu_{1} \cos \gamma_{1}}{V(x)} .
\end{aligned}
$$

From equation 11, the scattering angle $\gamma_{1}$ is derived in association with the scattering-azimuth $\gamma_{2}$ by the following relations:

$$
\begin{aligned}
& -\left.\frac{\partial z}{\partial h_{x}}\right|_{t, \mathbf{x}}=\tan \gamma_{1} \cos \gamma_{2}=-\frac{k_{h_{x}}}{k_{z}} ; \\
& -\left.\frac{\partial z}{\partial h_{y}}\right|_{t, \mathbf{x}}=\tan \gamma_{1} \sin \gamma_{2}=-\frac{k_{h_{y}}}{k_{z}},
\end{aligned}
$$

and the dip angle $\nu_{1}$ is derived in association with the dip-azimuth $\nu_{2}$ as

$$
\begin{gathered}
-\left.\frac{\partial z}{\partial x}\right|_{t, \mathbf{h}}=\tan \nu_{1} \cos \nu_{2}=-\frac{k_{x}}{k_{z}} ; \\
-\left.\frac{\partial z}{\partial y}\right|_{t, \mathbf{h}}=\tan \nu_{1} \sin \nu_{2}=-\frac{k_{y}}{k_{z}} .
\end{gathered}
$$

The decomposition of all four angle components of the angle domain (i.e., $\gamma_{1}, \gamma_{2}, \nu_{1}$, and $\nu_{2}$ ) in a similar manner to the proposal of Koren and Ravve (2011) and Ravve and Koren (2011), necessitate the computation of a 5D image. The practical challenge of computing so many image dimensions by wave-equation methods is highly expensive and tremendously time consuming. Therefore, we chose to focus our study in this paper on 2D only.

\section{IMAGE SPACE METHODS FOR ANGLE-DOMAIN DECOMPOSITION}

Fundamental relationships between the subsurface offset extended image and the angle-domain image are provided in equations 9 and 10. By following these relations, ADCIGs are in reach not only as a function of the scattering angles but also with respect to the dip angles. A suite of angle-domain decomposition techniques are formulated next for computing scattering-angle and dip-angle ADCIGs together with a hybrid multiangle ADCIG depending simultaneously on both angles. Although these techniques are derived here for the naive $2 \mathrm{D}$ case, the extension to the $3 \mathrm{D}$ case involves some additional steps to maintain the angle-domain azimuthal information, and requires further study.

The proposed postmigration techniques are applied in the extended image space and no longer involve the original seismic data. Notice that two angle axes are decomposed from a uniaxial subsurface offset extension. Hence, the extra dimension of the prestack image in the angle domain makes it more informative, without enforcing the remigration of the seismic data. Moreover, in addition to the forward angle decomposition, we also propose the inverse decomposition back to the subsurface offset domain. It allows the prestack image to be analyzed in both domains, back and forth, by simple postmigration transformations.

\section{Scattering-angle ADCIG decomposition}

Scattering-angle ADCIGs are decomposed according to the relationship with the subsurface offset extended image in equation 9. It includes the application of a classical Radon transform operator in the $z$ - $h$ domain, based on the angle-domain slope $p=\tan \gamma$ to guide the trajectory of integration. The Radon transform operator takes the form,

$$
A(x, z, p=\tan \gamma)=\int H(x, z+p h, h) d h,
$$


where $A$ and $H$ represents the angle and subsurface offset CIGs, respectively. Figure $7 \mathrm{a}$ illustrates the integration path of action this operator is following. Sava and Fomel (2003) derive an equivalent expression in the Fourier domain, which is more convenient for implementation.

By Fourier transform (indicated by the tilde symbol) over the z-axis, we obtain

$$
\tilde{A}\left(x, k_{z}, p\right)=\int \tilde{H}\left(x, k_{z}, h\right) e^{-i k_{z} p h} d h .
$$

Additional Fourier transform over the $h$-axis is recognized. Therefore, it is recast as

$$
\tilde{A}\left(x, k_{z}, p\right)=\tilde{\tilde{H}}\left(x, k_{z}, k_{h}\right),
$$

where $k_{h}=-p k_{z}$ is defined as the subsurface offset wavenumber. From equation 16, it is concluded that 1D Fourier transforms of scattering-angle ADCIGs are equivalent to the 2D Fourier transforms of the subsurface offset extended image, subject to the stretch of the $h$-axis according to the definition of $k_{h}$ (Sava and Fomel, 2003).

The inverse operator is formulated next to enable the transformation back to the subsurface offset domain. Equation 16 is rewritten as

$$
\begin{aligned}
\frac{1}{2 \pi} \int \tilde{A}\left(x, k_{z}, p\right) e^{i k_{z} p h} d p & =\frac{1}{2 \pi} \int \tilde{\tilde{H}}\left(x, k_{z}, k_{h}\right) e^{i k_{z} p h} d p \\
& =\frac{1}{\left|k_{z}\right|} \frac{1}{2 \pi} \int \tilde{\tilde{H}}\left(x, k_{z}, k_{h}\right) e^{i k_{h} h} d k_{h} .
\end{aligned}
$$

An inverse Fourier transform is recognized on the right side over $k_{h}$. Therefore, we obtain

$$
\left|k_{z}\right| \frac{1}{2 \pi} \int \tilde{A}\left(x, k_{z}, p\right) e^{i k_{z} p h} d p=\tilde{H}\left(x, k_{z}, h\right) .
$$

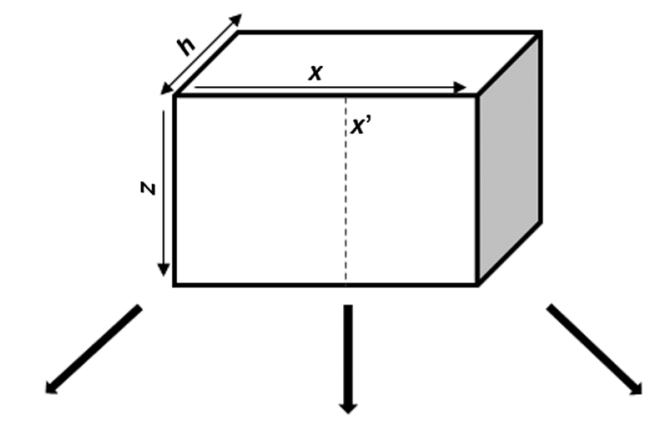

a)

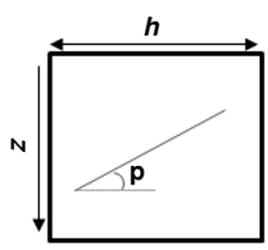

b)

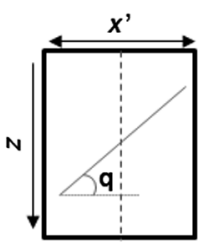

c)

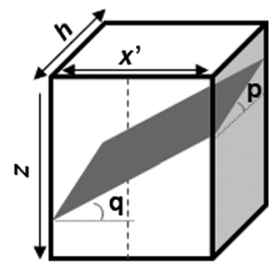

Figure 7. Radon transform path of integration, guided by the slopes $p$ and $q$. (a) Scattering-angle ADCIG, (b) dip-angle ADCIG, and (c) multiangle ADCIG are decomposed accordingly at the location $x^{\prime}$ marked with the dashed line.
Finally, by another inverse Fourier transform over $k_{z}$, we end up back in the subsurface offset domain:

$$
\operatorname{rho}(z) * \frac{1}{2 \pi} \int A(x, z-p h, p) d p=H(x, z, h),
$$

where rho $(z)$ stands for the inverse Fourier transform of the $\left|k_{z}\right|$ filter, which does not depend on the slope $p$, and the star symbol denotes convolution. Notice that the inverse of the original Radon transform in equation 14 turned to be another Radon transform subject to a filter and a change of sign.

\section{Dip-angle ADCIG decomposition}

Dip-angle ADCIGs are decomposed according to the relationship with the subsurface offset extended image in equation 10. As opposed to the scattering angles, here the decomposition is derived across the subsurface offset CIGs. It includes the application of a classical Radon transform operator in the $z-x$ domain for individual $h$ components, based on the angle-domain slope $q=\tan \nu$ to guide the trajectory of integration. The Radon transform operator takes the form,

$A(x, z, q=\tan \nu, h)=\frac{1}{\Delta x} \int_{-\Delta x / 2}^{\Delta x / 2} H\left(x+x^{\prime}, z+q x^{\prime}, h\right) d x^{\prime}$,

where $A$ and $H$ are the angle and subsurface offset CIGs, respectively. The additional variable $x^{\prime}$ represents the locality of the Radon transform around the $x$-coordinate; $\Delta x$ stands for the effective range, where $x^{\prime}$ is sampled. This is the local range across the image where dip-angle bins are being averaged. It is directly related to the resolution of the decomposed events in the dip domain: the resolution increases as $\Delta x$ gets larger. Usually, $\Delta x$ is in the order of hundreds of meters, depending on how steep or structurally complex the subsurface geology is. Notice that the decomposition in equation 20 is made for each subsurface offset independently as indicated by the additional fourth argument of the dip-angle ADCIGs. Figure $7 \mathrm{~b}$ illustrates the integration path of action that the operator in equation 20 is following.

Similar steps used to derive equation 15 are followed to obtain the 1D Fourier transform relation:

$$
\begin{aligned}
& \tilde{A}\left(x, k_{z}, q, h\right) \\
& =\frac{1}{\Delta x} \int_{-\Delta x / 2}^{\Delta x / 2} \tilde{H}\left(x+x^{\prime}, k_{z}, h\right) e^{-i k_{z} q x^{\prime}} d x^{\prime} .
\end{aligned}
$$

This relation can be recast by another Fourier transform, after extending the integration limits to infinity and introducing a boxcar function as follows:

$$
\begin{aligned}
& \tilde{A}\left(x, k_{z}, q, h\right) \\
& =\frac{1}{\Delta x} \int_{-\infty}^{\infty} \Pi\left(\frac{x^{\prime}}{\Delta x}\right) \tilde{H}\left(x+x^{\prime}, k_{z}, h\right) e^{-i k_{z} q x^{\prime}} d x^{\prime} .
\end{aligned}
$$


The additional Fourier transform is now recognized over $x^{\prime}$, and we obtain

$$
\tilde{A}\left(x, k_{z}, q, h\right)=\frac{1}{2 \pi} \sin c\left(k_{x} \Delta x\right) * \tilde{\tilde{H}}\left(k_{x}, k_{z}, h\right) e^{-i k_{x} x},
$$

where $k_{x}=-q k_{z}$ is defined as the horizontal wavenumber. The right side represents a convolution between a $\sin c$ function and the 2D Fourier transformed subsurface offset images multiplied by the exponential delay operator. Finally, the decomposition of the dip-angle ADCIGs is completed by a weighted averaging over all subsurface offsets, according to the weight function $W_{h}$ :

$$
\tilde{A}\left(x, k_{z}, q\right)=\frac{1}{2 \pi} \sum_{h} W_{h} \sin c\left(k_{x} \Delta x\right) * \tilde{\tilde{H}}\left(k_{x}, k_{z}, h\right) e^{-i k_{x} x}
$$

The weight function is usually designed to peak around the zero subsurface offset. However, the optimal design of this function, especially when the image is defocused due to migration velocity errors, is still an open question under study.

From equation 24, it is concluded that 1D Fourier transforms of dip-angle ADCIGs are equivalent to the average of the convolutional 2D Fourier transformed subsurface offset extended images, subject to the stretch of the $x$-axis according to the definition of $k_{x}$.

The inverse operator, transforming the image back to the subsurface offset domain is derived in a similar manner as in the previous section. Equation 23 is rewritten as

$$
\begin{aligned}
& \frac{1}{2 \pi} \int \tilde{A}\left(x, k_{z}, q, h\right) e^{i k_{z} q x^{\prime}} d q \\
& =\frac{1}{2 \pi} \int\left(\frac{1}{2 \pi} \sin c\left(k_{x} \Delta x\right) * \tilde{\tilde{H}}\left(k_{x}, k_{z}, h\right) e^{-i k_{x} x}\right) e^{i k_{z} q x^{\prime}} d q, \\
& =\frac{1}{\left|k_{z}\right|} \frac{1}{2 \pi} \int\left(\frac{1}{2 \pi} \sin c\left(k_{x} \Delta x\right) * \tilde{\tilde{H}}\left(k_{x}, k_{z}, h\right) e^{-i k_{x} x}\right) e^{-i k_{x} x^{\prime}} d k_{x} .
\end{aligned}
$$

An inverse Fourier transform is recognized on the right side over $k_{x}$. Therefore, we obtain

$$
\begin{aligned}
& \frac{1}{2 \pi} \int \tilde{A}\left(x, k_{z}, q, h\right) e^{i k_{z} q x^{\prime}} d q \\
& \quad=\frac{1}{\left|k_{z}\right|} \frac{1}{\Delta x} \Pi\left(\frac{x^{\prime}}{\Delta x}\right) \tilde{H}\left(x+x^{\prime}, k_{z}, h\right) .
\end{aligned}
$$

A stack operator over $x^{\prime}$ in the effective range $\Delta x$ is applied on both sides of the equation, after shifting the $x$-coordinate by $-x^{\prime}$ to obtain

$$
\begin{gathered}
\frac{1}{2 \pi} \sum_{-\Delta x / 2}^{\Delta x / 2} \int \tilde{A}\left(x-x^{\prime}, k_{z}, q, h\right) e^{i k_{z} q x^{\prime}} d q \\
\quad=\frac{1}{\left|k_{z}\right|} \sum_{-\Delta x / 2}^{\Delta x / 2} \frac{1}{\Delta x} \Pi\left(\frac{x^{\prime}}{\Delta x}\right) \tilde{H}\left(x, k_{z}, h\right) .
\end{gathered}
$$

The stack operator and the boxcar function cancel each other on the right side, which leads to the following expression:

$$
\left|k_{z}\right| \frac{1}{2 \pi} \sum_{-\Delta x / 2}^{\Delta x / 2} \int \tilde{A}\left(x-x^{\prime}, k_{z}, q, h\right) e^{i k_{z} q x^{\prime}} d q=\tilde{H}\left(x, k_{z}, h\right) .
$$

Finally, by another inverse Fourier transform over $k_{z}$, we end up back in the subsurface offset domain

$$
\operatorname{rho}(z) * \frac{1}{2 \pi} \sum_{-\Delta x / 2}^{\Delta x / 2} \int A\left(x-x^{\prime}, z-q x^{\prime}, q, h\right) d q=H(x, z, h) \text {, }
$$

where $\operatorname{rho}(z)$ is the same filter mentioned in the previous section. Once again, the inverse of the original Radon transform in equation 20 turned to be another Radon transform subject to a filter and a change of sign.

\section{Multiangle ADCIG decomposition}

It is quite remarkable that the steps that led to the dip-angle decomposition were made independent of the subsurface offset. As a result, the dip-angle ADCIG in equations 22 and 23 is still a function of the subsurface offset. Therefore, it can be further transformed to enable the additional decomposition of the scattering angles. The final hybrid angle-domain CIG represents the image by the dip and the scattering angles simultaneously and is considered here as a multiangle ADCIG. In relation to the classical Radon transform, the multiangle ADCIG is decomposed by a double Radon transform operator of the form,

$$
\begin{aligned}
& A(x, z, q=\tan \nu, p=\tan \gamma) \\
& \quad=\frac{1}{\Delta x} \int_{-\Delta x / 2}^{\Delta x / 2} \int H\left(x+x^{\prime}, z+q x^{\prime}+p h, h\right) d h d x^{\prime} .
\end{aligned}
$$

The path of integration involves a plane intersecting the subsurface offset extended image, defined by the slopes $q$ and $p$. Figure $7 \mathrm{c}$ illustrates this planar path of action this operator is following. Equation 30 is recast in the Fourier domain similar to the previous operators derived here. Combining the formulation of equations 22 and 15 leads to the 1D Fourier domain expression:

$$
\begin{aligned}
& \tilde{A}\left(x, k_{z}, q, p\right) \\
& \quad=\frac{1}{\Delta x} \iint \Pi\left(\frac{x^{\prime}}{\Delta x}\right) \tilde{H}\left(x+x^{\prime}, k_{z}, h\right) e^{-i k_{z}\left(q x^{\prime}+p h\right)} d h d x^{\prime} .
\end{aligned}
$$

Whereas combining equations 23 and 16 leads to the following expression, after recognizing the additional Fourier transforms over $x^{\prime}$ and $h$ on the right side

$$
\tilde{A}\left(x, k_{z}, q, p\right)=\frac{1}{2 \pi} \sin c\left(k_{x} \Delta x\right) * \tilde{\tilde{H}}\left(k_{x}, k_{z}, k_{h}\right) e^{-i k_{x} x} .
$$

One-dimensional Fourier transforms of the multiangle ADCIGs are equivalent to the convolutional 3D Fourier transforms of the subsurface offset extended image, subject to the stretch of $x$ - and $h$-axis according to the definition of $k_{x}$ and $k_{h}$. 
The inverse operator back to the subsurface offset domain is formulated from equation 32 by first recovering the subsurface offsets according to equation 18

$$
\begin{aligned}
& \left|k_{z}\right| \frac{1}{2 \pi} \int \tilde{A}\left(x, k_{z}, q, p\right) e^{i k_{z} p h} d p \\
& \quad=\frac{1}{2 \pi} \sin c\left(k_{x} \Delta x\right) * \tilde{\tilde{H}}\left(k_{x}, k_{z}, h\right) e^{-i k_{x} x},
\end{aligned}
$$

and then recovering the original $x$-axis in the same manner as in equation 28

$$
\begin{aligned}
& k_{z}^{2} \frac{1}{4 \pi} \sum_{-\Delta x / 2}^{\Delta x / 2} \iint \tilde{A}\left(x-x^{\prime}, k_{z}, q, p\right) e^{i k_{z}\left(q x^{\prime}+p h\right)} d p d q \\
& \quad=\tilde{H}\left(x, k_{z}, h\right) .
\end{aligned}
$$

The original image space is obtained on the right side by another inverse Fourier transform over $k_{z}$ :

$$
\begin{aligned}
& \mathrm{rho}^{2}(z) * \frac{1}{4 \pi} \sum_{-\Delta x / 2}^{\Delta x / 2} \iint A\left(x-x^{\prime}, z-q x^{\prime}-p h, q, p\right) d p d q \\
& =H(x, z, h) .
\end{aligned}
$$

Similar to the other two Radon operators formulated above, the inverse operator in equation 35 is also a Radon transform subject to a filter and a change of sign.

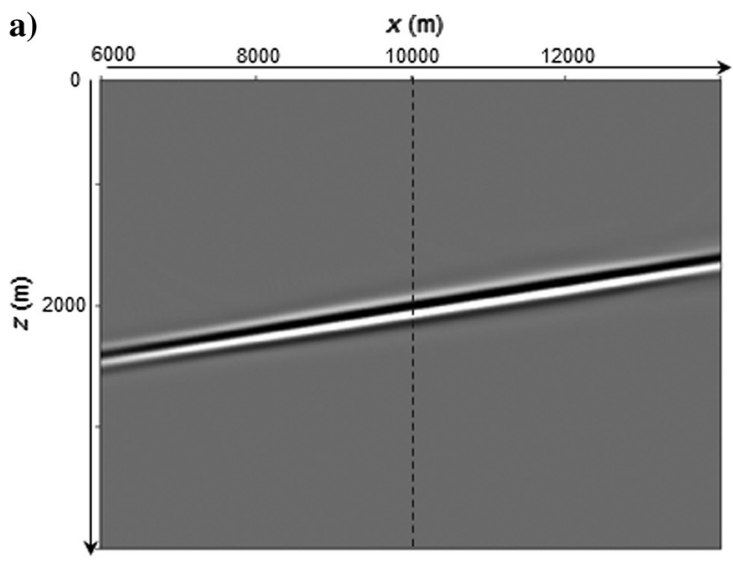

\section{SYNTHETIC DATA EXAMPLES}

The angle-domain decomposition techniques proposed above are exemplified here on three synthetic models. Because scattering-angle ADCIGs have been studied extensively in recent years, our main focus and discussion through this exemplification will involve the dip-angle domain decomposition. The synthetic data were computed by an acoustic Born modeling operator, which solves the perturbational wave equation by a fourth order in space and second order in a time finite-difference extrapolator. The modeling grid was sampled with a grid spacing of $10 \mathrm{~m}$ vertically and $25 \mathrm{~m}$ horizontally, and with a $4 \mathrm{~ms}$ time sample increment. Trace data were computed for 161 shots located on the surface, starting from $6000 \mathrm{~m}$ with spacing of $50 \mathrm{~m}$. The 401 towed receivers were evenly spread in front and behind each shot with $25 \mathrm{~m}$ spacing (split-spread geometry). A Ricker wavelet with $8 \mathrm{~Hz}$ dominant frequency was used as a source. For imaging, an extended Born-type migration operator (Symes, 2008) was applied, where the subsurface offset extension was sampled with $25 \mathrm{~m}$ of grid spacing. True migration velocity was used in all examples, unless otherwise stated.

\section{Dip-domain decomposition - Where have the response "tails" gone?}

Our first example is the simplistic model consisting of a single $-5^{\circ}$ dipping interface between two homogeneous layers. Figure 8a presents the imaging results by showing the zero subsurface offset image on the left, and one of the subsurface offset CIGs on the right. The CIG was calculated at the horizontal position marked with the

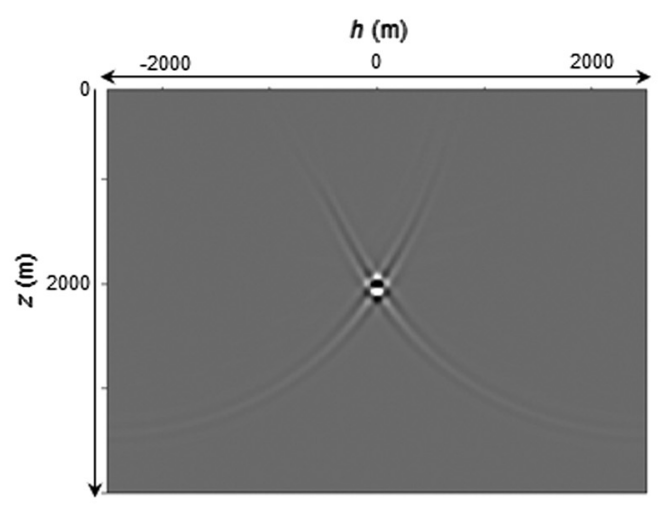

b)

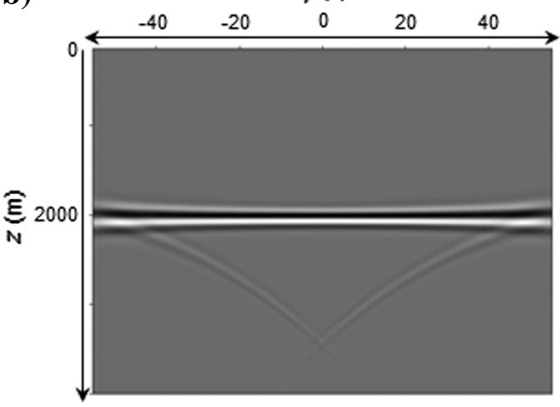

c)

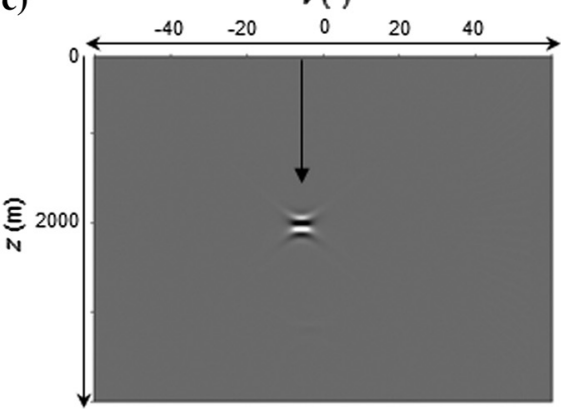

d)

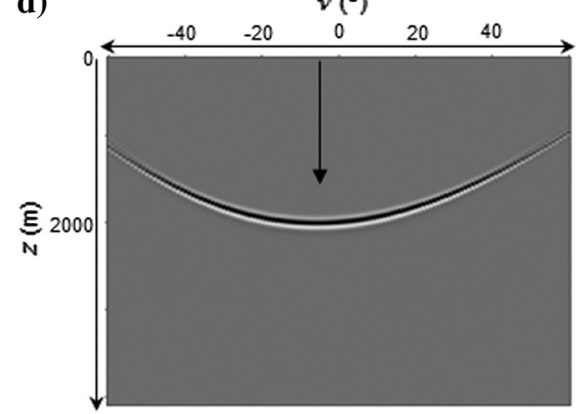

Figure 8. Imaging of a $-5^{\circ}$ dipping reflector. (a) The zero subsurface offset image on the left to a subsurface offset CIG, (b) scattering-angle ADCIG, (c) dip-angle ADCIG, and (d) dip-angle ADCIG generated by Kirchhoff migration. All CIGs were calculated at the location marked with the dashed line. 
dashed line. The reflection image is focused at the zero subsurface offset trace, although some energy is clearly leaking to nonzero offsets. This smearing artifact is related to the truncation of the acquisition geometry by the maximum acquisition offset as described by Mulder (2014). The angle-domain decomposition techniques, introduced in the previous section, have been used to calculate the scattering-angle ADCIG in Figure 8b and the dip-angle ADCIG in Figure $8 \mathrm{c}$ for the same marked horizontal position. Both ADCIGs were decomposed by using the same subsurface offset CIGs presented in Figure 8a. The scattering-angle ADCIG shows the typical flat appearance at the true depth of reflection $(2000 \mathrm{~m}$ in this example), implying that the correct migration velocity was used. Notice that the subsurface offset domain smearing artifact was transformed into two diffraction curves peaking at the maximum angle of illumination. The reflectivity in the dip-angle ADCIG in Figure $8 \mathrm{c}$ is prominently focused at the reflection depth, indicating the specular dip angle of the subsurface reflector $\left(-5^{\circ}\right.$ in this example) on the gather axis as shown by the arrow.

For comparison, we remigrated the seismic data by an angle-domain Kirchhoff migration to calculate an equivalent dip-angle ADCIG for the same subsurface model. The Kirchhoff dip-angle ADCIG is presented in Figure 8d, and shows a concave response having its stationary point at the specular dip angle of reflection (marked with the arrow). This concave response is what dip-domain studies, involving Kirchhoff migration, usually observe and report. Among them, Landa et al. (2008) describe the response a dipping reflector has in the dip domain as a smile-like curve, and they derive its corresponding analytic formula in a homogeneous medium accordingly. Koren and Ravve (2010) provide the 3D extension of that formula with respect to the dip angle and the dip-azimuth. This well-studied response differs from our focused dip-domain observation in Figure 8c. Although the same specular dip information is provided in both cases, our observation is missing the tails of the concave response away from the specular point. These tails are usually related to aliasing noise generated by the migration's diffraction stack operator due to incomplete destructive interference. Many studies were aimed to reduce these tails by restricting the migration aperture to include mostly the specular energy, or by filtering the image in the dip domain (Chen, 2004; Bienati et al., 2009; Dafni and Reshef, 2014).

Figure 9 demonstrates the mechanism responsible for the concave reflection response in the dip domain, by again describing prestack migration as a superposition of impulse responses made by individual data traces. On the left, the interaction between three elliptic impulse responses (see equation 4) in the image space is illustrated in blue, to constructively generate an image of a dipping up reflector denoted by the black line. Dip-angle ADCIGs like the one presented in Figure 8d, are decomposed before the imaging condition is applied. Therefore, they capture some nonspecular but slightly constructive energy (indicated by the red triangle) in addition to the strong specular information provided at the reflector (indicated by the red circle). On the right side of the figure, a dip-angle ADCIG is formed by mapping the nonspecular information (i.e., the red triangle) to the tails of the smile-like response, and the specular event (i.e., the red circle) to the apex position at the stationary point. The dip-angle decomposition proposed in this study, takes place after the imaging condition in the subsurface offset domain. In such a case, the artificial nonspecular energy is destructively summed, and the tails of the response are annihilated. What's left in the gather is only the essential specular spot as shown in Figure 8c. To support this claim, the seismic data were remigrated once again to the subsurface offset domain, this time by using an extended Kirchhoff migration operator. Our goal was to prove that the same "tailless" dip-angle response is obtained even by using Kirchhoff migration methods. The fact that the nonspecular aliasing artifact is lost is an attribute of the subsurface offset extension. It has to do with the behavior of the image in this domain, and not the nature of the migration method. The extended Kirchhoff migration was formulated by extending the general integral kernel to the subsurface offset domain. It takes the form,

$$
\begin{aligned}
I(\mathbf{x}, \mathbf{h})= & \int d \mathbf{x}_{\mathbf{r}} \int d \mathbf{x}_{\mathbf{s}} A_{r}\left(\mathbf{x}+\mathbf{h}, \mathbf{x}_{\mathbf{r}}\right) A_{s}\left(\mathbf{x}-\mathbf{h}, \mathbf{x}_{s}\right) \\
& \times D\left(\mathbf{x}_{\mathbf{r}}, \tau\left(\mathbf{x}+\mathbf{h}, \mathbf{x}_{\mathbf{r}}\right)+\tau\left(\mathbf{x}-\mathbf{h}, \mathbf{x}_{s}\right) ; \mathbf{x}_{\mathbf{s}}\right)
\end{aligned}
$$

where $A_{s}$ and $A_{r}$ are the amplitudes of the ray-theoretic approximation of the shot and receiver Green's functions.

The Kirchhoff migration results are shown in Figure 10a. The zero subsurface offset image section is provided on the left to a subsurface offset CIG, calculated at the same marked horizontal location. Except for minor amplitude variations, the subsurface offset image results obtained by Kirchhoff migration are similar to those computed by the wave-equation migration. In Figure 10b, the corresponding dip-angle ADCIG is decomposed. It shows the same specular spot at the reflector's depth as observed in Figure 8b. There is no evidence whatsoever of the nonspecular tails of a concave response. We find this spot-like response in the dip domain remarkably promising. Not only that dip-angle ADCIGs can be said now in the same sentence with wave-equation migration, our decomposition method can better explain the response of seismic reflections in the dip domain, and it can provide more reliable and artifact-free information about the structure of the earth.

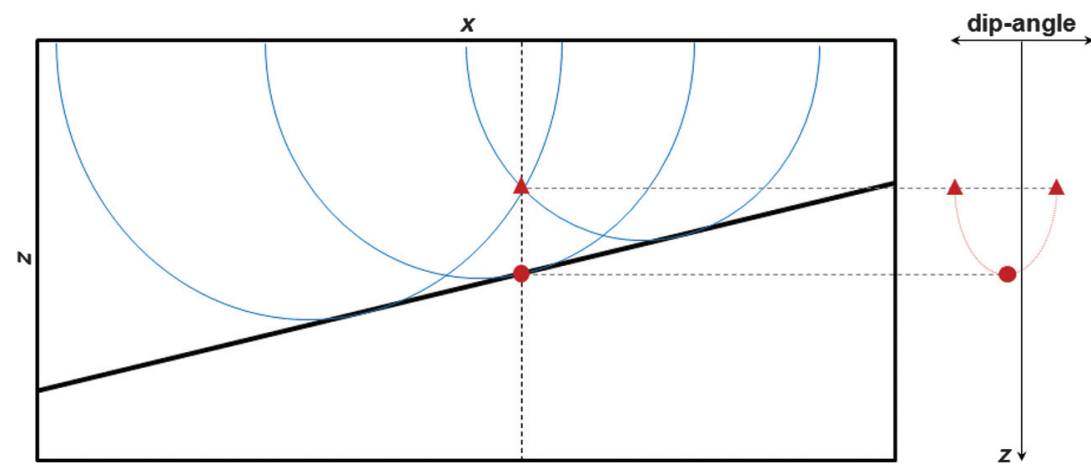

Figure 9. The mechanism of the concave response associated with reflections in the dip domain. The interaction between the migration of individual data traces (impulse responses) constructively maps energy of specular reflections (the red circle) to the dip domain on the right. Slightly constructive energy is mapped as well by nonspecular interaction (the red triangle). 


\section{Multiangle decomposition}

Multiangle ADCIGs were decomposed for the first example's two-layer model, by the double Radon transform operator introduced in the previous section. Figure 11a presents the multiangle gather generated at the horizontal location marked in Figure $8 \mathrm{a}$. This gather has two angle axes that show simultaneously the scattering and the dip angles for each depth point. Therefore, a 3D view is shown in the figure, by projecting the gather along each axis. The familiar flat image is clearly recognized in the scattering-angle projection at the bottom-left corner, whereas a specular spot is focused at the $-5^{\circ}$ dip trace in the dip-angle projection at the bottom-right corner. The multiangle ADCIG is offering a convenient platform to analyze each depth point individually by both angles. The best way to display this information is by projecting depth slices from the gather. The top-left corner of the 3D image in Figure 11a shows the depth projection extracted from the reflector's depth of $2000 \mathrm{~m}$. At this depth level, a coherent strip pattern is observed, where all the scattering angles respond in-phase at the same specular dip angle of $-5^{\circ}$. This strip pattern has been introduced by Dafni and Reshef (2012), although it was in the content of Kirchhoff migration methods. According to their approach, the analysis of individual depth projections related to the imaging depth of the subsurface reflectors may assist to improve the image quality and the migration velocity assurance. In areas where the subsurface geology is complex, simultaneous analysis of the image by two angle axes might reveal the essential information needed to explain it better.

When velocity errors are present, the indication of a coherent strip pattern in the multiangle depth projection is lost. It is demonstrated in Figure 11b, where we rerun the migration by using $10 \%$ too-high migration velocity, and we redecomposed the multiangle ADCIG. As expected, the scattering-angle projection at the bottomleft corner of the figure shows a curved-down event due to the sign of the velocity error. The specular spot in the dip-angle projection at the bottom-right corner became slightly unfocused and blurry, and it is wrongly placed in depth. In the depth projection at the upper corner, an "open" pattern has been revealed, by combining the nonflatness and the blurring effects observed along the two angle axes. As the scattering angle increases, the signal spreads out of the specular dip position and wrong dip angles are imaged. Also note that this depth projection was extracted at wrong imaging depth of $2200 \mathrm{~m}$. The "open" pattern, associated with too-fast migration velocity, was reported similarly by Dafni and Reshef (2012), in the content of Kirchhoff migration methods.
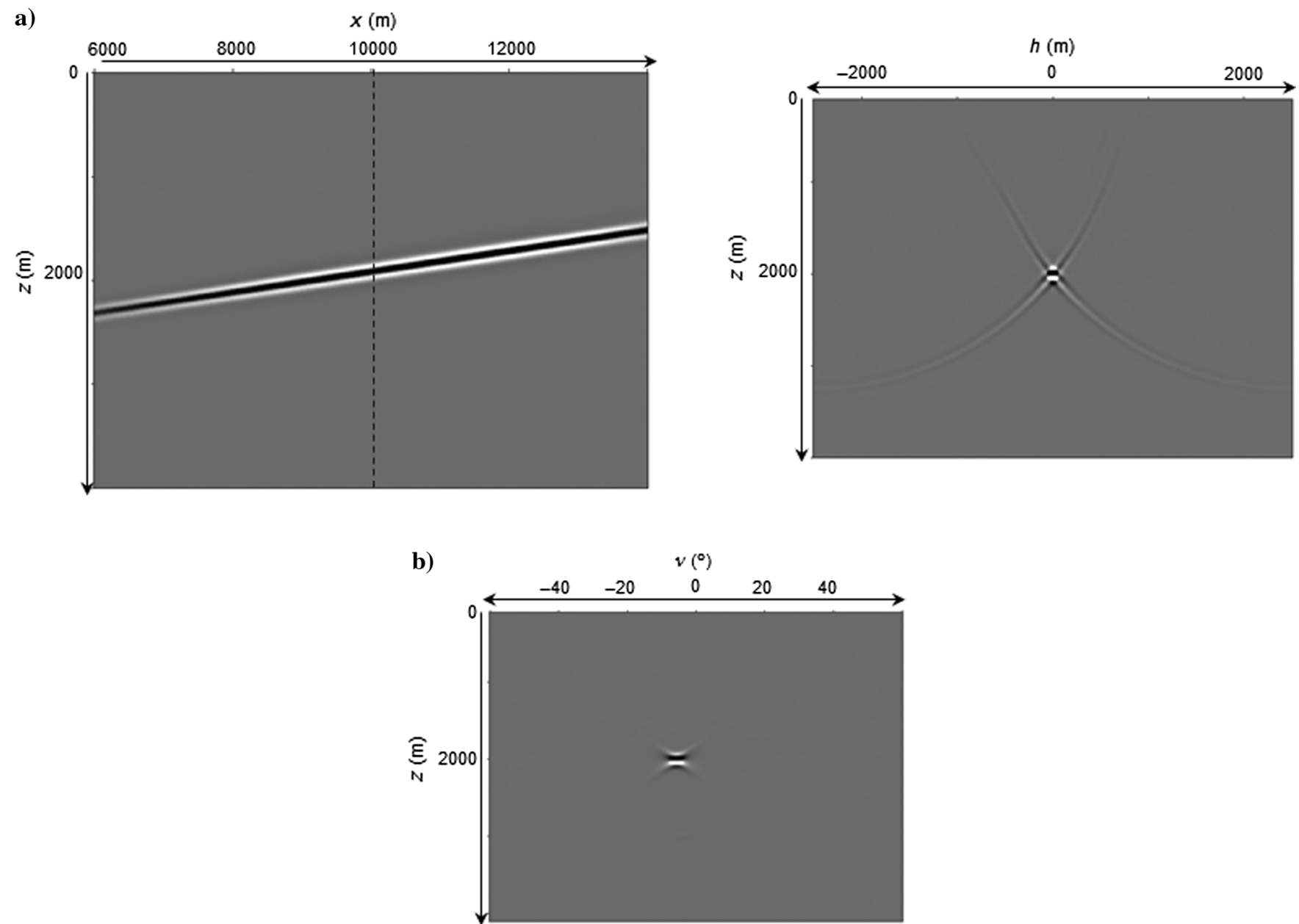

Figure 10. Imaging of a $-5^{\circ}$ dipping reflector by an extended Kirchhoff migration in the subsurface offset domain. (a) The zero subsurface offset image on the left to a subsurface offset CIG, and (b) dip-angle ADCIG. CIGs were calculated at the location marked with the dashed line. 

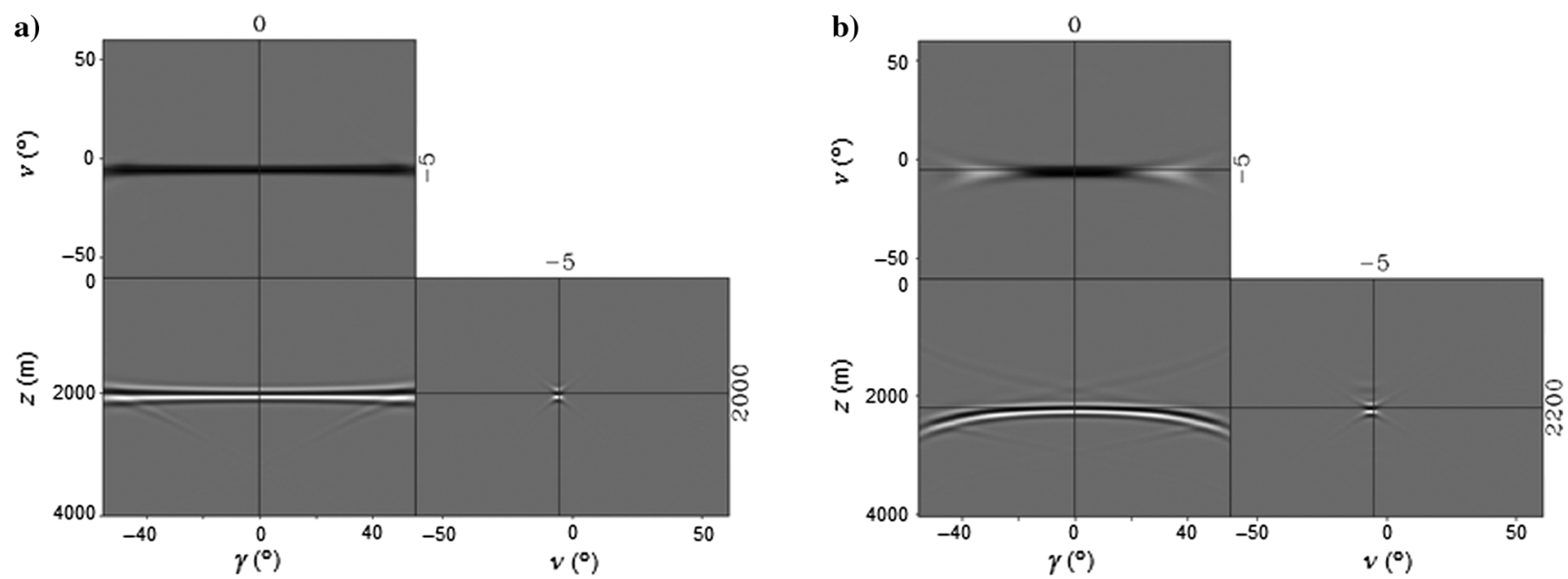

Figure 11. Multiangle ADCIG decomposed by (a) correct and (b) 10\% too-high migration velocity, at the location marked with the dashed line in Figure 8a. Scattering-angle projection and dip-angle projection are shown at the bottom left and right corners, respectively. A depth projection is provided at the top left corner, extracted from the reflector's imaging depth.
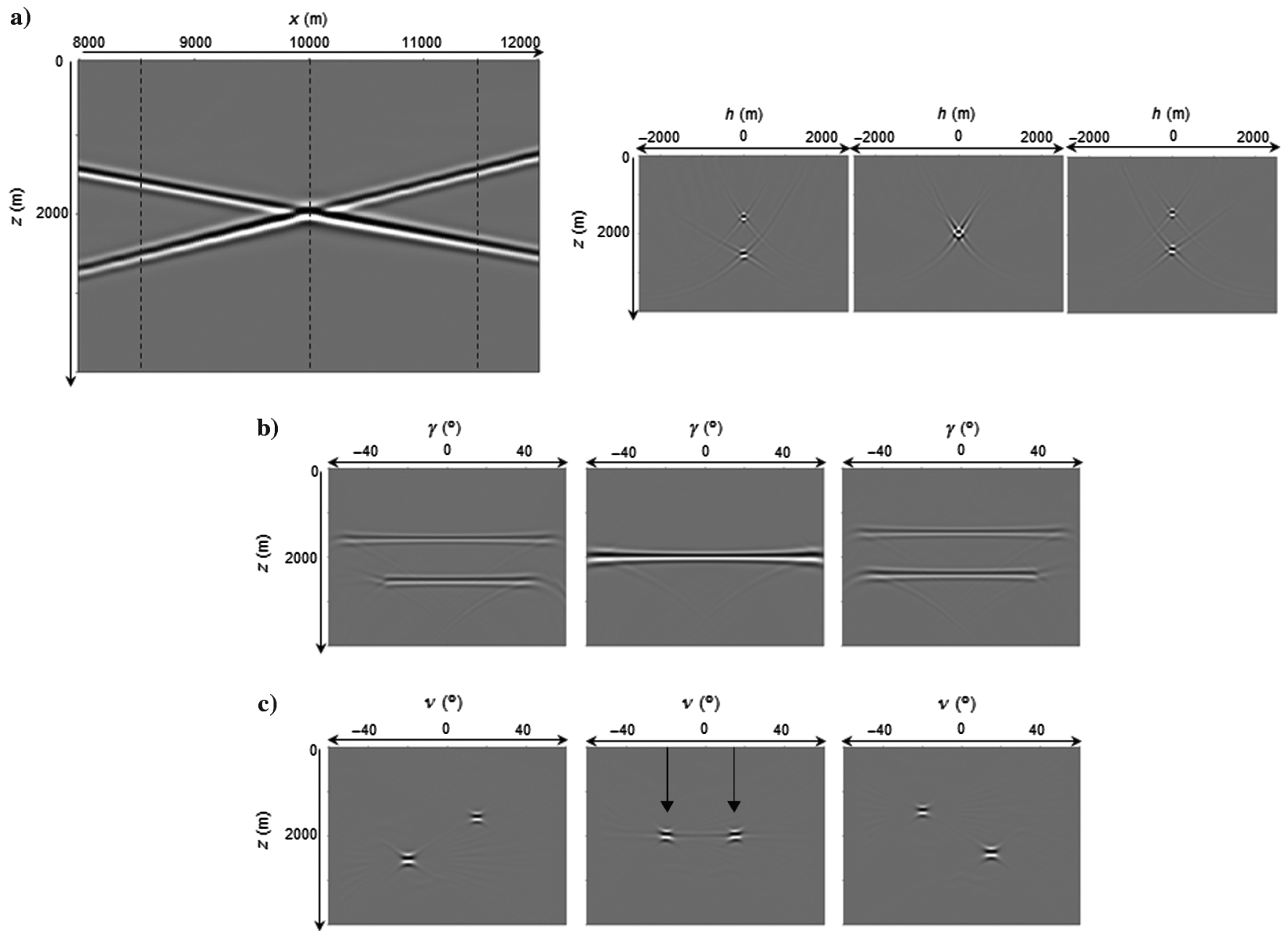

Figure 12. Conflicting dips scenario. (a) The zero subsurface offset image on the left to three subsurface offset CIGs, (b) scattering-angle ADCIGs, and (c) dip-angle ADCIGs. CIGs were calculated at the locations marked with the dashed lines in (a). 


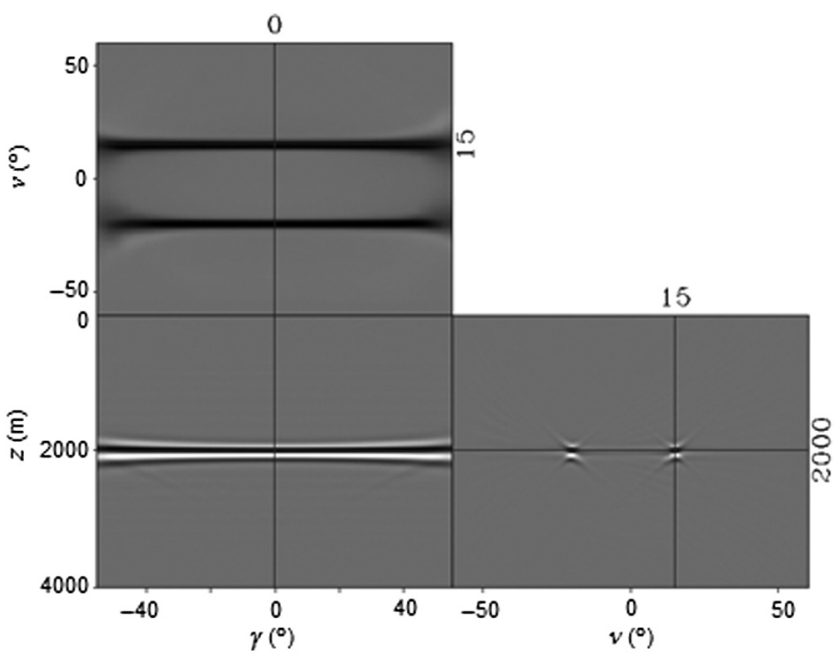

Figure 13. Multiangle ADCIG decomposed at the conflicting location $x=10,000 \mathrm{~m}$. Scattering-angle projection and dip-angle projection are shown at the bottom left and right corners, respectively. A depth projection is shown at the top left corner, extracted from the conflicting depth of $2000 \mathrm{~m}$.

\section{Conflicting-dips scenario}

In the next synthetic example, two dipping reflectors $\left(15^{\circ}\right.$ and $\left.-20^{\circ}\right)$ are present. CIGs calculated at the horizontal location, where the two reflectors intersect $(x=10,000 \mathrm{~m})$, represent the problematic scenario of conflicting dips. The imaging results of this example are summarized in Figures 12 and 13. The zero subsurface offset image and three subsurface offset CIGs are shown on the left and right sides of Figure 12a, respectively. The dashed lines mark the location where the CIGs were calculated. The CIG in the middle represents the conflicting dips situation, in which only a single event is noticed. This loss of information could lead to incorrect CIG analysis. For example during velocity analysis, velocity errors might be related to a single event and ignore the other one. The other two CIGs in Figure 12a were calculated off the conflicting location and show the two dipping events focused at zero subsurface offset. Scattering-angle ADCIGs are decomposed in Figure 12b. The same problematic situation is present in this domain as well, where it is almost impossible to distinguish between the two dipping events at the conflicting location. The only way to differentiate between the conflicting events is by a structural criterion, such as the one the dip domain provides. Figure $12 \mathrm{c}$ presents the dip-angle ADCIGs,
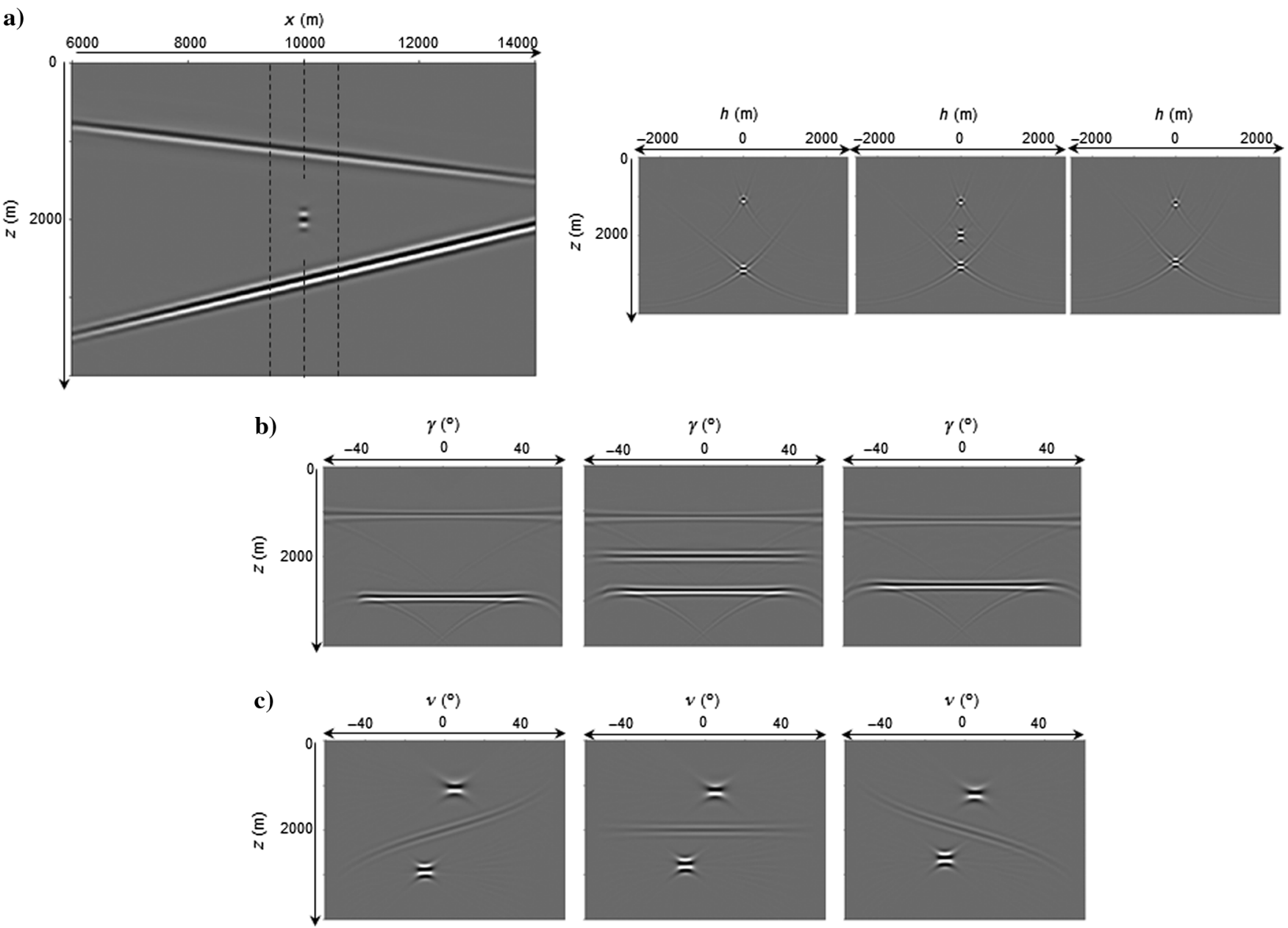

Figure 14. Imaging of two dipping reflectors $\left(5^{\circ}\right.$ and $-10^{\circ}$ dip angles) and a point diffractor. (a) The zero subsurface offset image on the left to three subsurface offset CIGs, (b) scattering-angle ADCIGs, and (c) dip-angle ADCIGs. CIGs were calculated at the locations marked with the dashed lines in (a). 
a)
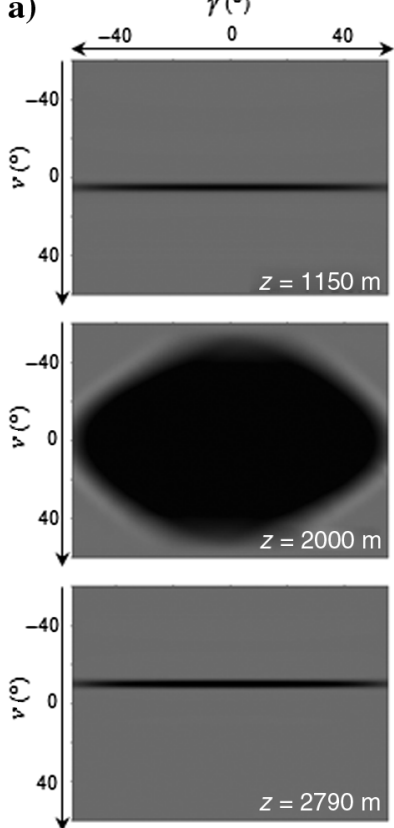

b)
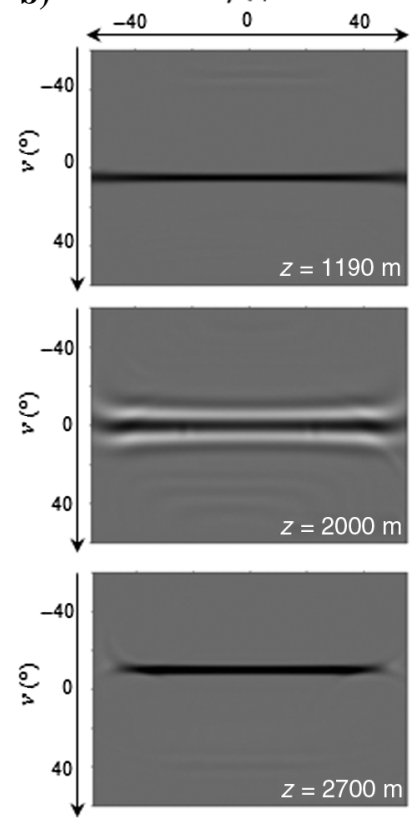

Figure 15. Multiangle ADCIGs decomposition. Three multiangle depth projections were extracted (a) at the point diffractor location $(x=10,000 \mathrm{~m})$ and (b) $500 \mathrm{~m}$ to the right of this location. The depth of each projection is labeled in white at the bottom.

decomposed at the same three horizontal locations. Two separated events are clearly shown even in the middle gather, representing the conflicting location. The two arrows indicate that the events are focused around the two specular dip angles of $15^{\circ}$ and $-20^{\circ}$.

Because dip information is also available by the multiangle ADCIG, it enables the separation between conflicting events in a similar manner. The multiangle ADCIG, decomposed at the conflicting location, is presented by the 3D display in Figure 13. The differentiation between the events is noticed in the dip-angle projection by the two coherent spots and in the depth projection, taken at the conflicting depth of $2000 \mathrm{~m}$. The depth projection shows two elongated strips at the $15^{\circ}$ and $-20^{\circ}$ dip angles.

\section{Seismic diffractions}

Our last synthetic example involves the imaging of seismic diffractions. The subsurface model consists of a local heterogeneity at the depth of $2000 \mathrm{~m}$, considered here as a point diffractor. The scattered energy from this small-scale object generates a diffraction event in the acquired seismic data. Two dipping reflectors are also present in the model above and below the diffractor. These reflectors are dipping $5^{\circ}$ down and $-10^{\circ}$ up, respectively. The zero subsurface offset image is presented on the left side of Figure 14a. The CIGs in this example were calculated at the diffractor location and $500 \mathrm{~m}$ off to each side (see the dashed markers embedded in the image). On the right side of Figure 14a, the subsurface offset CIGs are displayed. Notice that while observing seismic diffractions from
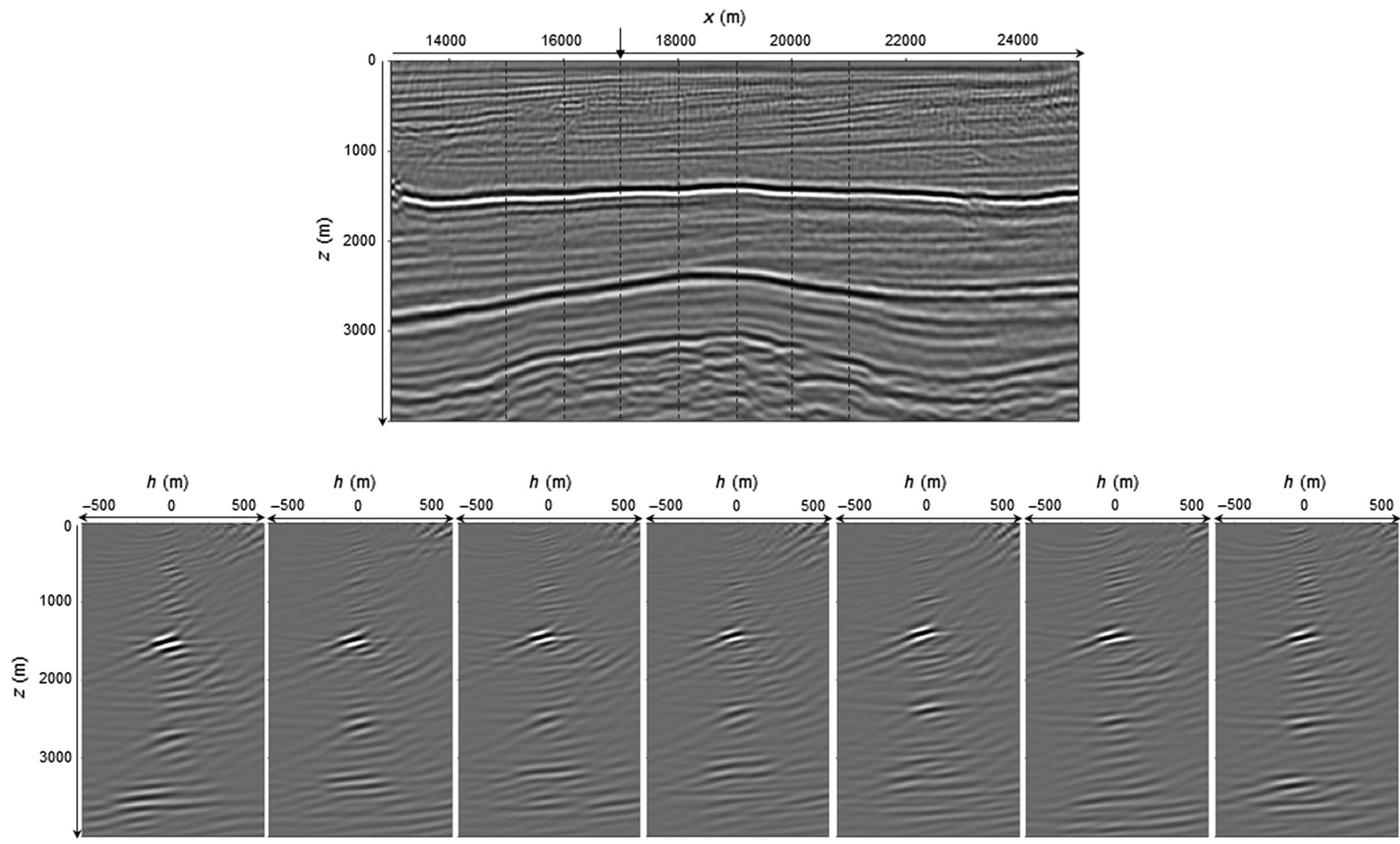

Figure 16. Field data example. The zero subsurface offset image on top of seven subsurface offset CIGs, calculated at the locations marked with the dashed lines. 
above (the middle gather in the figure), they respond in the same way as seismic reflections in this domain: they are focused at the zero offset trace. However, when observed from the side, their response is smeared and weakened as the distance between the CIG location and the diffractor increase, until it vanishes completely from the gather. It is almost impossible to recognize the diffraction event in the left and right CIGs in Figure 14a.

The scattering-angle ADCIGs are decomposed in Figure 14b. The gather in the middle was calculated above the diffractor and shows the same response similarity between reflections and diffractions (both are flat in this domain). Nevertheless, the diffraction event is absent in the other two gathers on the sides because they were calculated too far away from its origin. Seismic diffractions are known by their unique response in the dip domain. They are distinguished from reflections and have a flat appearance when the migration velocity is correct and when the gather is observed directly above the diffraction location (Landa et al., 2008; Klokov and Fomel, 2012). Figure 14c presents the dip-angle ADCIGs decomposed in this example. The flat-shaped diffraction and the reflections focused spots are easily recognized in the middle gather. Notice that the diffraction event is still present in the gathers distant $500 \mathrm{~m}$ away from the diffractor location. It has an inclined shape with an opposite direction of inclination to the left and to the right sides of the diffractor. This prominent and unique response, even far away from the diffraction origin, makes the analysis of diffractions in the dip domain a natural choice. Moreover, seismic diffractions were revealed as highly sensitive to velocity errors in the dip domain, which may improve the resolution of the migration velocity estimation during velocity analysis (Reshef and Landa, 2009).
As expected, seismic diffractions have a distinguished response in the multiangle ADCIGs as well. In Figure 15, we present a set of depth projections extracted from two multiangle ADCIGs, calculated directly above (Figure 15a) and to the right (Figure 15b) of the diffraction position. The depth of each projection is labeled in white. The coherent strip patterns of the reflection events are recognized in Figure 15a and 15b above and below the diffraction depth of $2000 \mathrm{~m}$. They indicate the $5^{\circ}$ and $-10^{\circ}$ specular dip values, respectively. The $2000 \mathrm{~m}$ depth projection in Figure 15a was calculated exactly at the $z$ - $x$-coordinates of the point diffractor. Therefore, it reveals an image related to the fundamental migration diffraction stack operator, which is usually constituted in the basis of angle-domain imaging methods. All scattering and dip angles respond in-phase at this depth, forming a coherent and planar pattern bounded by the maximum angles of illumination on both axes. The same depth level is observed by the middle projection in Figure $15 \mathrm{~b}$, which was calculated $500 \mathrm{~m}$ to the right of the diffraction. Because the horizontal location does not coincide with the one of the diffraction, a different pattern is shown in this projection. It is elongated out-of-phase parallel to the scattering-angle axis at zero dip.

\section{FIELD DATA EXAMPLE}

We apply our angle decomposition methods next to a field data set acquired off shore the east Mediterranean Sea. The data were collected along a 2D line by a towed streamer having 240 geophone channels and maximum offset of $6106 \mathrm{~m}$. The subsurface offset extended image was constructed by a Born-type migration operator, as
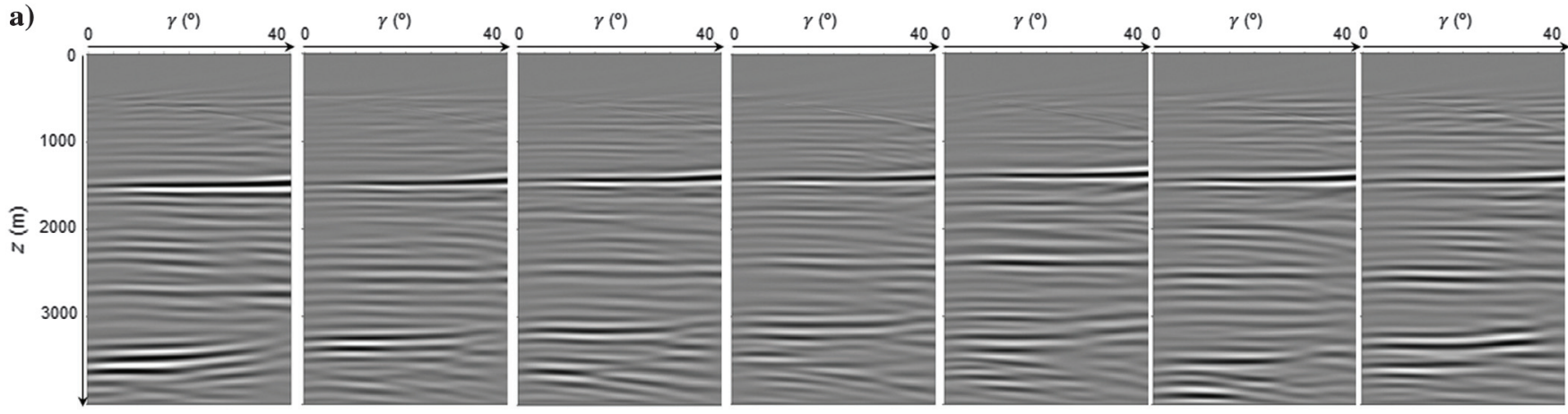

b)
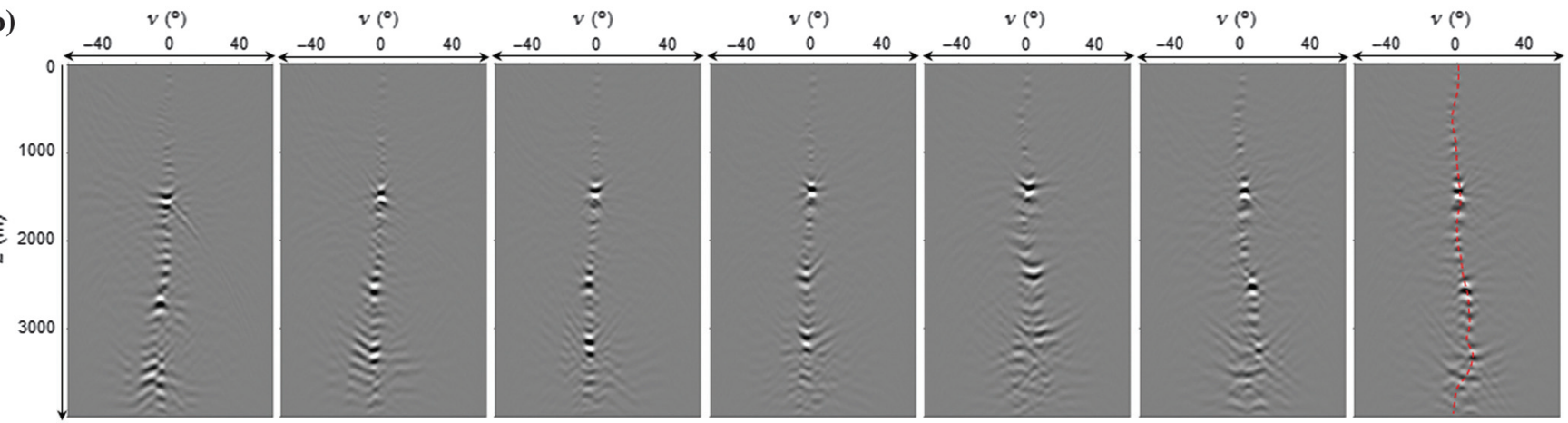

Figure 17. Field data example. Decomposition of (a) scattering-angle ADCIGs and (b) dip-angle ADCIGs at the marked locations in Figure 16. The extraction of a dip profile by following the specular spots is demonstrated with the red curve in the rightmost dip-angle ADCIG. 
mentioned in the previous section. The extended image is presented in Figure 16. The zero subsurface offset image section is shown on top. Two prominent key horizons are identified approximately at depths of 1500 and $2700 \mathrm{~m}$ across the image. A third horizon is also noticed below, although it appears broken and with poor quality. At the bottom part of Figure 16, seven subsurface offset CIGs are presented. These image gathers were calculated at the locations marked with the dashed lines. The first two key horizons are focused nicely around the zero offset trace, implying that an accurate migration velocity was used. Notice that the amplitude polarity corresponding to the second horizon is reversed, due to a velocity reversal in the vertical direction. However, the third reflection event is slightly defocused because the migration velocity is not as optimized below the second horizon.

ADCIGs were calculated at the same marked locations. They are presented in Figures 17 and 18. The scattering-angle ADCIGs in Figure 17a show fairly flat events, although some poor quality remnant moveout is still present in the deep part of the image. Because single-ended spread was used to acquire the data (all geophones towed behind the seismic source), only positive scattering angles were decomposed. The dip-angle ADCIGs in Figure 17b show the same specular spot response associated with reflections in this

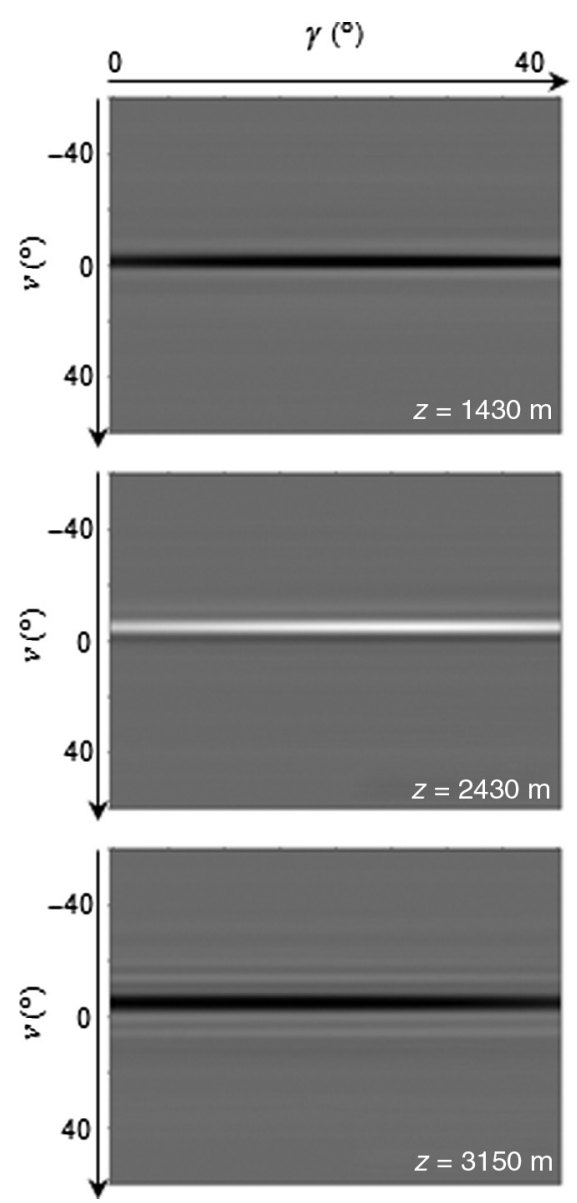

Figure 18. Field data example. Multiangle ADCIG decomposition at the location marked with an arrow in Figure 16. Three multiangle depth projections were extracted from the depth of the key horizons (labeled at the bottom in white). study. They provide priceless information with regard to the directivity of the subsurface geology. By picking the dominant dipdomain spot-like events, a dip profile is defined at each horizontal location (demonstrated by the red curve in the rightmost gather). The focusing of the dip-domain events is weakened in the deep part of the image. It is negatively affected by the nonoptimized migration velocity and the poor signal. The events are smeared, which makes the extraction of a well-defined specular dip direction less certain at this part of the image.

The seismic reflections observed in this data set show the same generic strip pattern in the multiangle ADCIGs as discussed in detail through the synthetic exemplification in the previous section. Figure 18 presents three multiangle ADCIG depth projections, calculated at the location marked with an arrow in Figure 16. The projections were taken from the depth of the three key horizons (labeled in white). Sharp strip patterns are clearly noticed in the figure. Their position along the dip-angle axis indicates a mildly dipping up geology (small and negative dip angles) at this horizontal location. The polarity reversal at the depth of the second key horizon is observed in the middle depth projection, as the strip pattern response has an opposite sign of amplitude (white color).

\section{CONCLUSION}

Angle-domain decomposition techniques were introduced for prestack wave-equation migration methods. A suite of Radon transform operators was proposed to enable the calculation of scatteringangle, dip-angle, and multiangle ADCIGs from the postmigration subsurface offset extended image. The inverse operator of each decomposition technique was derived as well, which makes the transform from the subsurface offset domain to the angle domain a reversible process. Because the subsurface offset constitutes as the building blocks constructing the angle domain in this study, some new insights regarding the behavior of the image at finite-subsurface offset were presented. It was derived from the approach describing prestack migration as a superposition of subsurface offset extended impulse responses, made by individual data traces.

Dip-angle ADCIGs are traditionally associated as an attribute of Kirchhoff migration methods. This work alleviates this restriction by allowing wave-equation migration methods to decompose dipdomain images as well. A unique dip-domain response of seismic reflections was discovered by using the proposed decomposition technique. It shows a spot-like response, focused at the reflector's depth and indicating its specular dip on the dip-angle axis. In contrast, Kirchhoff migration of reflections usually generates a concave response instead of a spot, although the same essential structural information is provided at the stationary point. This remarkable contradiction is quite unusual because two methods, aimed to decompose the same image, give significantly different results. It was clarified by emphasizing that this study's techniques take place after the migration's extended imaging condition is applied. The extension of the image to the subsurface offset domain before the angles are decomposed naturally annihilates nonspecular contributions to the image as those related to the tails of the concave response. A spot-like response, rather than concave, was obtained by decomposing dip-angle ADCIGs from a subsurface offset extended Kirchhoff migration image to strengthen this thesis. Hence, not only that the dip domain is promoted to be another extension wave-equation migration might have, it provides a focused-in dip image involving the specular contribution solely without artificial tails. 
Scattering and dip angles were decomposed simultaneously in the formulation of the multiangle ADCIG, showing two angle axes for each image point. This hybrid image represents the inner kernel of the classical angle-domain diffraction stack operator. When the velocity model is known, the image point associated with a subsurface reflector shows a coherent strip pattern focused around the well-defined specular dip angle, whereas a point diffractor shows a planar pattern where all angles are diffracted equally.

The decomposition of the dip angles aside from the scattering angles, as two sets of ADCIGs or as a single multiangle ADCIG, increases the sensitivity to artifacts and noise while analyzing the postmigration image. We believe it may produce the added value needed for calculating more reliable reflectivity models, and accelerate wave-equation inversion methods such as full-waveform inversion.

\section{ACKNOWLEDGMENTS}

We are grateful to the sponsors of the Rice University Inversion Project for their long-term support, in particular to Shell International Exploration and Production Inc. for its financial support. We also acknowledge the Israeli Ministry of National Infrastructures, Energy and Water Resources for partial financial support, as part of the Graduate Fellowship Program of postdoctoral researchers at leading universities abroad in the fields of science and engineering of natural gas and oil. We wish to thank S. Fomel for the many discussions and cooperation contributing to the development of this paper. We have benefited greatly from the high-performance computing resources provided by the Rice University Research Computing Support Group.

\section{APPENDIX A}

\section{SUBSURFACE OFFSET EXTENDED IMPULSE RESPONSE}

The response of the extended migration operator, presented in equation 1 , to a single data trace is considered here as an extended impulse response in the subsurface offset domain.

In the following, the kinematics of this response will be studied analytically, assuming a known and homogeneous background medium.

The subsurface offset extended migration operator, as presented in equation 1:

$$
\begin{aligned}
I(\mathbf{x}, \mathbf{h})= & \int d \mathbf{x}_{\mathbf{r}} \int d \mathbf{x}_{\mathbf{s}} \int d t \frac{\partial^{2}}{\partial t^{2}} D\left(\mathbf{x}_{\mathbf{r}}, t ; \mathbf{x}_{\mathbf{s}}\right) \\
& \times \int d \tau G\left(\mathbf{x}+\mathbf{h}, t-\tau ; \mathbf{x}_{\mathbf{r}}\right) G\left(\mathbf{x}-\mathbf{h}, \tau ; \mathbf{x}_{\mathbf{s}}\right) .
\end{aligned}
$$

Because this operator is applied here on a single data trace, the integration over the shot and receiver coordinates can be ignored, by setting the Green's function coordinates to honor the shot-receiver location on the acquisition surface $\left(\mathbf{x}_{\mathbf{r}}, \mathbf{x}_{\mathbf{s}}\right)$ :

$$
\begin{aligned}
I(\mathbf{x}, \mathbf{h}) & =\int d t \frac{\partial^{2}}{\partial t^{2}} D\left(\mathbf{x}_{\mathbf{r}}, t ; \mathbf{x}_{\mathbf{s}}\right) \\
& \times \int d \tau G\left(\mathbf{x}-\mathbf{x}_{\mathbf{r}}+\mathbf{h}, t-\tau\right) G\left(\mathbf{x}-\mathbf{x}_{\mathbf{s}}-\mathbf{h}, \tau\right) .
\end{aligned}
$$

The data trace is represented here by a delta-function shifted to the reflection's time $t_{s r}$ :

$$
D\left(\mathbf{x}_{\mathbf{r}}, t ; \mathbf{x}_{\mathbf{s}}\right)=\delta\left(t-t_{s r}\right) .
$$

Substituting this into equation A-2

$$
\begin{aligned}
I(\mathbf{x}, \mathbf{h}) & =\int d t \frac{\partial^{2}}{\partial t^{2}} \delta\left(t-t_{s r}\right) \\
& \times \int d \tau G\left(\mathbf{x}-\mathbf{x}_{\mathbf{r}}+\mathbf{h}, t-\tau\right) G\left(\mathbf{x}-\mathbf{x}_{\mathbf{s}}-\mathbf{h}, \tau\right),
\end{aligned}
$$

and integrating over time $t$, we obtain

$$
I(\mathbf{x}, \mathbf{h})=\int d \tau \frac{\partial^{2}}{\partial t^{2}} G\left(\mathbf{x}-\mathbf{x}_{\mathbf{r}}+\mathbf{h}, t_{s r}-\tau\right) G\left(\mathbf{x}-\mathbf{x}_{\mathbf{s}}-\mathbf{h}, \tau\right) .
$$

The Green's function has a singularity at the time $t_{s r}$, and so do all of its time derivatives. The time derivatives represent the same kinematics as the Green's function itself. Because only the kinematic properties of the impulse response are under study, we ignore the time derivatives is equation A-5. It makes the formulation simpler and reveals the same kinematic behavior. Equation A-5 is rewritten accordingly as

$$
I(\mathbf{x}, \mathbf{h})=\int d \tau G\left(\mathbf{x}-\mathbf{x}_{\mathbf{r}}+\mathbf{h}, t_{s r}-\tau\right) G\left(\mathbf{x}-\mathbf{x}_{\mathbf{s}}-\mathbf{h}, \tau\right) .
$$

Because a delta-function reflection was assumed in constant velocity $V$ medium, we use a whole space uniform velocity Green's function of the form

$$
G(\mathbf{x}, t)=\frac{\delta\left(t-\frac{|\mathbf{x}|}{V}\right)}{|\mathbf{x}|}
$$

Incorporating this choice of Green's function into equation A-6 yields

$$
I(\mathbf{x}, \mathbf{h})=\int d \tau \frac{\delta\left(t_{s r}-\tau-\frac{\left|\mathbf{x}-\mathbf{x}_{\mathbf{r}}+\mathbf{h}\right|}{V}\right)}{\left|\mathbf{x}-\mathbf{x}_{\mathbf{r}}+\mathbf{h}\right|} \frac{\delta\left(\tau-\frac{\left|\mathbf{x}-\mathbf{x}_{\mathbf{s}}-\mathbf{h}\right|}{V}\right)}{\left|\mathbf{x}-\mathbf{x}_{\mathbf{s}}-\mathbf{h}\right|}
$$

and integrating over migration time $\tau$, we obtain

$$
I(\mathbf{x}, \mathbf{h})=\frac{\delta\left(t_{s r}-\frac{\left|\mathbf{x}-\mathbf{x}_{\mathbf{r}}+\mathbf{h}\right|+\left|\mathbf{x}-\mathbf{x}_{\mathbf{s}}-\mathbf{h}\right|}{V}\right)}{\left|\mathbf{x}-\mathbf{x}_{\mathbf{r}}+\mathbf{h}\right|\left|\mathbf{x}-\mathbf{x}_{\mathbf{s}}-\mathbf{h}\right|} .
$$

The shot-receiver coordinates on the acquisition surface satisfies the following relations with respect to the acquisition half-offset $\mathbf{H}$ and the midpoint location $\mathbf{x}_{\mathbf{m}}$ :

$$
\mathbf{x}_{\mathbf{s}}=\mathbf{x}_{m}-\mathbf{H} ; \quad \mathbf{x}_{\mathbf{r}}=\mathbf{x}_{m}+\mathbf{H}
$$


Rewriting equation A-9 accordingly takes the form

$$
I(\mathbf{x}, \mathbf{h})=\frac{\delta\left(t_{s r}-\frac{\left|\left(\mathbf{x}-\mathbf{x}_{m}\right)+(\mathbf{h}-\mathbf{H})\right|+\left|\left(\mathbf{x}-\mathbf{x}_{m}\right)-(\mathbf{h}-\mathbf{H})\right|}{V}\right)}{\left|\left(\mathbf{x}-\mathbf{x}_{m}\right)+(\mathbf{h}-\mathbf{H})\right|\left|\left(\mathbf{x}-\mathbf{x}_{m}\right)-(\mathbf{h}-\mathbf{H})\right|} .
$$

Hence, the image will be constructed according to the argument of the delta function in equation A-11, which defines the imaging condition as

$$
t_{s r}=\frac{\left|\left(\mathbf{x}-\mathbf{x}_{m}\right)+(\mathbf{h}-\mathbf{H})\right|+\left|\left(\mathbf{x}-\mathbf{x}_{m}\right)-(\mathbf{h}-\mathbf{H})\right|}{V} .
$$

In the general 3D case and for given acquisition and subsurface offsets, this condition represents an ellipsoid in the image space of the form

$$
\frac{\left(x-x_{m}\right)^{2}}{\left(\frac{1}{2} V t_{s r}\right)^{2}}+\frac{\left(y-y_{m}\right)^{2}}{\left(\frac{1}{2} V t_{s r}\right)^{2}}+\frac{z^{2}}{\left(\frac{1}{2} V t_{s r}\right)^{2}-(\mathbf{h}-\mathbf{H})^{2}}=1 .
$$

The ellipsoid in equation A-13 is an extended isochron surface of constant traveltime $t_{s r}$.

Equation A-13 is rewritten by substituting the traveltime according to the hyperbolic relation $\frac{1}{2} V t_{s r}=\sqrt{z_{0}^{2}+\mathbf{H}^{2}}$ :

$$
\frac{\left(x-x_{m}\right)^{2}}{\left(z_{0}^{2}+\mathbf{H}^{2}\right)}+\frac{\left(y-y_{m}\right)^{2}}{\left(z_{0}^{2}+\mathbf{H}^{2}\right)}+\frac{z^{2}}{\left(z_{0}^{2}+\mathbf{H}^{2}\right)-(\mathbf{h}-\mathbf{H})^{2}}=1,
$$

where $z_{o}$ is the zero-dip imaging depth of the seismic event or the bottommost point of the ellipsoid. Note that the focal distance of the ellipsoid is defined by the difference $|\mathbf{h}-\mathbf{H}|$. Therefore, only when the subsurface offset is set to zero $(\mathbf{h}=0)$, the focal points will represent the shot-receiver coordinates on the acquisition surface $\left(\mathbf{x}_{\mathbf{r}}, \mathbf{x}_{\mathbf{s}}\right)$. In any other nonzero case, the focal points are shifted.

Equation 2 in the body of the paper was written according to equation A-14 for the 2D case. It is important to emphasize again that any amplitude effects related to the impulse response of the extended migration operator are excluded here, and they are outside the scope of this paper.

\section{REFERENCES}

Audebert, F., L. Nicoletis, P. Froidevaux, and H. Rakotoarisoa, 2003, True amplitude migration in the angle domain by regularization of illumination: 73rd Annual International Meeting, SEG, Expanded Abstracts, 921-924.

Bartana, A., D. Kosloff, and I. Ravve, 2006, Discussion and reply on "Angle-domain common-image gathers by wavefield continuation methods" (Paul C. Sava and Sergey Fomel, 2003, Geophysics, 68, 1065-1074): Geophysics, 71, no. 1, X1-X3, doi: 10.1190/1.2168013.

Beylkin, G., 1985, Imaging of discontinuities in the inverse scattering problem by inversion of a causal generalized Radon transform: Journal of Mathematical Physics, 26, 99-108, doi: 10.1063/1.526755.

Bienati, N., C. Andreoletti, F. Perrone, V. Lipari, and M. Giboli, 2009, Limited aperture migration in the angle domain: 71st Annual International Conference and Exhibition, EAGE, Extended Abstracts, U005.

Biondi, B., and W. W Symes, 2004, Angle-domain common-image gathers for migration velocity analysis by wavefield-continuation imaging: Geophysics, 69, 1283-1298, doi: 10.1190/1.1801945.
Biondi, B., and T. Tisserant, 2004, 3D angle-domain common-image gathers for migration velocity analysis: Geophysical Prospecting, 52, 575-591, doi: 10.1111/j.1365-2478.2004.00444.x.

Bleistein, N., 1987, On the imaging of reflectors in the earth: Geophysics, 52, 931-942, doi: 10.1190/1.1442363.

Brandsberg-Dahl, S., M. V. de Hoop, and B. Ursin, 2003, Focusing in dip and AVA compensation on scattering-angle/azimuth common image gathers: Geophysics, 68, 232-254, doi: 10.1190/1.1543210.

Browaeys, T. J., 2008, Dip-angle common-image gathers by wave-equation migration: 78th Annual International Meeting, SEG, Expanded Abstracts, 3003-3007.

Chen, J., 2004, Specular ray parameter extraction and stationary-phase migration: Geophysics, 69, 249-256, doi: 10.1190/1.1649392.

Claerbout, J. F., 1985, Imaging the earth's interior: Blackwell Scientific Publishing.

Dafni, R., and M. Reshef, 2012, Interval velocity analysis using multiparameter common image gathers: Geophysics, 77, no. 4, U63-U72, doi: 10 $.1190 /$ geo2011-0403.1.

Dafni, R., and M. Reshef, 2014, Image enhancement by multi-parameter characterization of common image gathers: Geophysical Prospecting, 62, 1453-1467, doi: 10.1111/1365-2478.12155.

Dafni, R., and M. Reshef, 2015, Multidip tomography formulation for migration velocity analysis: Geophysics, 80, no. 2, U1-U12, doi: 10.1190/ geo2014-0285.1.

de Bruin, C., C. Wapenaar, and A. Berkhout, 1990, Angle-dependent reflectivity by means of prestack migration: Geophysics, 55, 1223-1234, doi: $10.1190 / 1.1442938$

Jin, H., G. A. McMechan, and H. Guan, 2014, Comparison of methods for extracting ADCIGs from RTM: Geophysics, 79, no. 3, S89-S103, doi: 10 1190/geo2013-0336.1.

Klokov, A., and S. Fomel, 2012, Separation and imaging of seismic diffractions using migrated dip-angle gathers: Geophysics, 77, no. 6, S131S143, doi: $10.1190 /$ geo2012-0017.1.

Koren, Z., and I. Ravve, 2010, Specular/diffraction imaging by full azimuth subsurface angle domain decomposition: 80th Annual International Meeting, SEG, Expanded Abstracts, 3268-3272.

Koren, Z., and I. Ravve, 2011, Full-azimuth subsurface angle domain wavefield decomposition and imaging. Part 1: Directional and reflection image gathers: Geophysics, 76, no. 1, S1-S13, doi: 10.1190/1.3511352.

Landa, E., S. Fomel, and M. Reshef, 2008, Separation, imaging and velocity analysis of seismic diffractions using migrated dip-angle gathers: 78th Annual International Meeting, SEG, Expanded Abstracts, 21762180.

Li, Z., B. Tang, and S. Ji, 2012, Subsalt illumination analysis using RTM 3D dip gathers: 82nd Annual International Meeting, SEG, Expanded Abstracts, $1-6$.

Miller, D., M. Oristaglio, and G. Beylkin, 1987, A new slant on seismic imaging: Migration and integral geometry: Geophysics, 52, 943-964, doi: $10.1190 / 1.1442364$.

Montel, J. P., and G. Lambare, 2013, Wave equation angle domain common image gather asymptotic analysis: 83rd Annual International Meeting, SEG, Expanded Abstracts, 3757-3761.

Mosher, C., and D. Foster, 2000, Common angle imaging condition for prestack depth migration: 70th Annual International Meeting, SEG, Expanded Abstracts, 830-833.

Mulder, W. A., 2014, Subsurface offset behavior in velocity analysis with extended reflectivity images: Geophysical Prospecting, 62, 17-33, doi: 10 $.1111 / 1365-2478.12073$.

Nolan, C. J., and W. W. Symes, 1996, Imaging and coherency in complex structure: 66th Annual International Meeting, SEG, Expanded Abstracts, 359-363.

Prucha, M., B. Biondi, and W. W. Symes, 1999, Angle-domain commonimage gathers by wave-equation migration: 69th Annual International Meeting, SEG, Expanded Abstracts, 824-827.

Ravve, I., and Z. Koren, 2011, Full-azimuth subsurface angle domain wavefield decomposition and imaging. Part 2: Local angle domain: Geophysics, 76, no. 2, S51-S64, doi: 10.1190/1.3549742.

Reshef, M., and A. Rueger, 2008, Influence of structural dip angles on interval velocity analysis: Geophysics, 73, no. 4, U13-U18, doi: 10.1190/1 .2918741 .

Reshef, M., and E. Landa, 2009, Post-stack velocity analysis in the dip-angle domain using diffractions: Geophysical Prospecting, 57, 811-821, doi: 10 $.1111 / j .1365-2478.2008 .00773 . x$.

Rickett, j. E., and P. C. Sava, 2002, Offset and angle-domain common image-point gathers for shot-profile migration: Geophysics, 67, 883-889, doi: $10.1190 / 1.1484531$

Sava, P. C., and S. Fomel, 2003, Angle-domain common-image gathers by wavefield continuation methods: Geophysics, 68, 1065-1074, doi: 10 $.1190 / 1.1581078$.

Sava, P. C., and I. Vasconcelos, 2011, Extended imaging conditions for wave-equation migration: Geophysical Prospecting, 59, 35-55, doi: 10 $.1111 / \mathrm{j} .1365-2478.2010 .00888 . x$. 
Stolk, C. C., and W. W. Symes, 2004, Kinematic artifacts in prestack depth migration: Geophysics, 69, 562-575, doi: 10.1190/1.1707076.

Stolk, C. C., M. V. de Hoop, and W. W. Symes, 2009, Kinematics of shotgeophone migration: Geophysics, 74, no. 6, WCA19-WCA34, doi: 10 $.1190 / 1.3256285$

Symes, W. W., 2008, Migration velocity analysis and waveform inversion: Geophysical Prospecting, 56, 765-790, doi: 10.1111/j.1365-2478.2008.00698.x.

ten Kroode, A. P. E., D. J. Smit, and A. R. Verdel, 1994, Linearized inverse scattering in the presence of caustic: The International Society for Optical Engineering, SPIE, Expanded Abstracts, 28-42.

Wu, R. S., and L. Chen, 2003, Prestack depth migration in the angledomain using beamlet decomposition: Local image matrix and local
AVA: 73rd Annual International Meeting, SEG, Expanded Abstracts, 973-976.

Wu, R. S., and L. Chen, 2006, Directional illumination analysis using beamlet decomposition and propagation: Geophysics, 71, no. 4, S147-S159, doi: $10.1190 / 1.2204963$.

Xu, S., H. Chauris, G. Lambaré, and M. S. Noble, 2001, Common-angle migration: A strategy for imaging complex media: Geophysics, 66, 1877-1894, doi: 10.1190/1.1487131.

Yilmaz, O., 2001, Seismic data analysis: SEG.

Yoon, K., and K. J. Marfurt, 2006, Reverse-time migration using Poynting vector: Exploration Geophysics, 37, 102-107, doi: 10.1071/ EG06102. 